\title{
Solar System Events at High Spatial Resolution
}

D. Gavel, B. Macintosh, C. E. Max, S. G. Gibbard, I. de Pater,

A. M. Ghez, E. F. Young, C. P. McKay, K. H. Baines

February 19, 1999

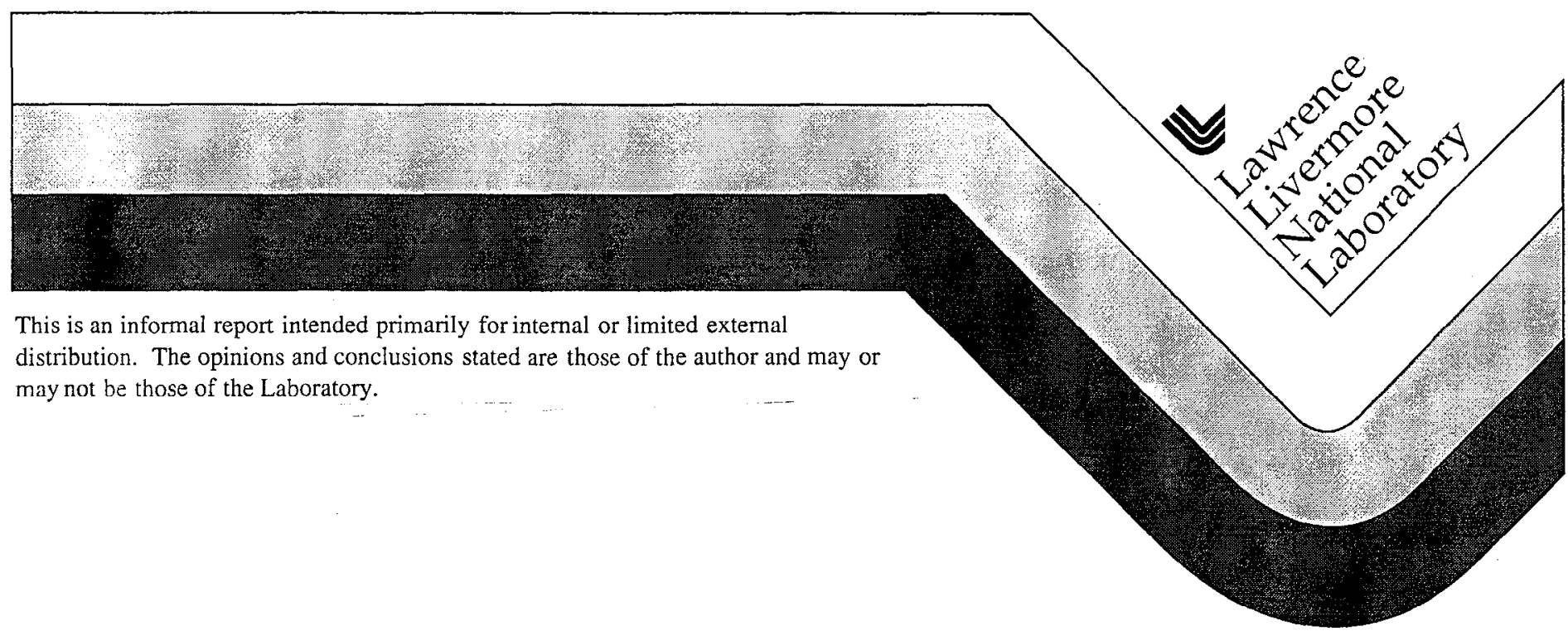




\section{DISCLAIMER}

This document was prepared as an account of work sponsored by an agency of the United States Government. Neither the United States Government nor the University of California nor any of their employees, makes any warranty, express or implied, or assumes any legal liability or responsibility for the accuracy, completeness, or usefulness of any information, apparatus, product, or process disclosed, or represents that its use would not infringe privately owned rights. Reference herein to any specific commercial product, process, or service by trade name, trademark, manufacturer, or otherwise, does not necessarily constitute or imply its endorsement, recommendation, or favoring by the United States Government or the University of California. The views and opinions of authors expressed herein do not necessarily state or reflect those of the United States Government or the University of California, and shall not be used for advertising or product endorsement purposes.

This report has been reproduced directly from the best available copy.

Available to DOE and DOE contractors from the Office of Scientific and Technical Information

P.O. Box 62, Oak Ridge, TN 37831

Prices available from (615) 576-8401, FTS 626-8401

Available to the public from the

National Technical Information Service

U.S. Department of Commerce

5285 Port Royal Rd.,

Springfield, VA 22161 


\title{
SOLAR SYSTEM EVENTS AT HIGH SPATIAL RESOLUTION \\ LDRD FINAL REPORT \\ 96-ERI-002
}

October, 1995 September, 1998

D. Gavel ${ }^{1}$, Principal Investigator

Collaborators:

B. Macintosh ${ }^{2}$, C.E. Max $^{2}$, S.G. Gibbard ${ }^{2}$

I. de Pater ${ }^{3}$, A.M.Ghez ${ }^{4}$,

E.F. Young ${ }^{5}$, C.P. McKay ${ }^{6}$, and K.H Baines ${ }^{7}$

\footnotetext{
${ }^{1}$ Laser Program, Lawrence Livermore National Laboratory

${ }^{2}$ Institute of Geophysics and Planetary Physics,

Lawrence Livermore National Laboratory

3 Dept. of Astronomy,University of California at Berkeley

${ }^{4}$ Dept. of Physics, University of California at Los Angeles

${ }^{5}$ Southwest Research Institute, Boulder, Colorado

${ }^{6}$ NASA Ames Research Center, Moffett Field, California

7 Jet Propulsion Laboratory, Pasadena, California
}

\begin{abstract}
Until relatively recent advances in technology, astronomical observations from the ground were limited in image resolution by the blurring effects of earth's atmosphere. The blur extent, ranging typically from 0.5 to 2 seconds of arc at the best astronomical sights, precluded ground-based observations of the details of the solar system's moons, asteroids, and outermost planets. With the maturing of a high resolution image processing technique called speckle imaging the resolution limitation of the atmosphere can now be largely overcome. Over the past three years we have used speckle imaging to observe Titan, a moon of Saturn with an atmospheric density comparable to Earth's, Io, the volcanically active innermost moon of Jupiter, and Neptune, a gas giant outer planet which has continually changing planet-encircling storms. These observations were made at the world's largest telescope, the Keck telescope in Hawaii and represent the highest resolution infrared images of these objects ever taken.
\end{abstract}

AUSPICES: This research was performed under the auspices of the U.S. Department of Energy by Lawrence Livermore National Laboratory under Contract W-7405-ENG-48. 


\section{INTRODUCTION: Project Goals and Accomplishments}

High-spatial-resolution observations of planets, asteroids, and comets have traditionally been the exclusive domain of space-based telescopes. The Voyager space probe missions, the Galileo spacecraft (presently orbiting Jupiter), and the Hubble Space Telescope (HST) have all given us unique views of solar system objects. However, spacecraft fly-bys are unique, while the highly over subscribed HST can allocate time to planet imaging only once or twice a year. The goal of this LDRD project is to make extensive use of speckle interferometry at the world's largest telescope, the Keck 10 meter Telescope on Mauna Kea in Hawaii, to attain very high resolution images of several solar system bodies at infrared wavelengths.

Until relatively recent advances in technology, astronomical observations from the ground were limited in spatial resolution by the blurring effects of earth's atmosphere. The blur extent, ranging typically from 0.5 to 2 seconds of arc at the best astronomical sights, precluded ground-based observations of the details of the solar system's moons, asteroids, and outermost planets. Many major observatories, including Keck, are procuring adaptive optics systems that will largely overcome the resolution limit of the atmosphere. The physical limit of spatial resolution, the so called diffraction-limit, is inversely dependent on telescope diameter, and for Keck, this can be typically be ten times smaller than the atmospheric blur.

In advance of adaptive optics at Keck, (first light is in February, 1999) we have, over the past three years under the auspices of this LDRD project, been using speckle interferometry to attain diffraction-limited reconstructed images of solar system objects. This technique uses many short $(\sim 100 \mathrm{~ms})$ exposure images coupled with extensive post-processing to form a single high resolution image. The approach uses a larger amount of telescope time than an equivalent long exposure image because of the overhead in handling the increased data of many short exposures, and is also limited to bright astronomical targets because each short exposure data must overcome noise in instrument readout, but the technique is useful neverthcless for a number of scientifically intercsting targcts, particularly the bright moons and outer planets we have been looking at.

Titan, the largest moon of Saturn, subtends an angle of less than one second of arc when viewed from Earth. It has a relatively thick atmosphere which is, like Earth, composed of $80 \%$ nitrogen. This atmosphere has no oxygen however but has a considerable amount of hydrocarbon aerosols (methane, ethane, and other carbon chain molecules). According to current Titan atmospheric chemistry models, methane in the upper atmosphere photolyzes into longer chain hydrocarbons; the long chain hydrocarbons form even more complex hydrocarbon molecules, condense into globules, and rain onto the surface. The surface therefore is thought to be covered in a hydrocarbon goo but perhaps also has lakes of liquid ethane with dissolved methane which interact with and restore the ethane to the atmosphere.

The hydrocarbon acrosols in the upper atmosphere crcatc a thick smog that completely obscures the moon's surface at visible wavelengths. The cameras of the Voyager spacecraft 
missions saw Titan only as a hazy orange ball. However, at certain near infrared wavelength bands Titan's atmosphere is transparent and the surface is revealed.

Our Keck speckle imaging observations show Titan's surface at a resolution of 0.02 arcseconds per pixel (each pixel representing about $150 \mathrm{~km}$ on the surface) with 50 pixels across the disk. At such resolution, Titan can be seen to have continent sized bright regions, perhaps highlands of rock and ice, and very black areas which are quite possibly pools of liquid hydrocarbons. Over the past three years, we have mapped most of the surface in $2 \mu \mathrm{m}$ wavelength band, and considerable portions in the 1.6 and $1.1 \mu \mathrm{m}$ bands. These maps, which represent the surface reflectivity to incident sunlight at these wavelengths, yield information about the surface composition.

Earth's early atmosphere originally had a high hydrocarbon content and no oxygen, like Titan, prior to the formation of life, so there is considerable interest in this moon as an example of prebiotic Earth. Such enthusiasm must be tempered by the fact that the temperature on Titan's surface is 100 Kelvins, much colder than that of early Earth and much colder than any known life form can survive.

Neptune is so distant from the sun that active, fast changing, storm systems come as a surprise. The enormous energy in these storms cannot be derived from the sun's incident radiation; instead it must come from planet's internal heat, caused by gravity and nuclear processes in the core. We have observed Neptune over several planet rotations and tracked the storm features, characterizing their sizes, locations, and velocities.

Neptune's atmosphere is mostly Hydrogen, with trace amounts of methane and other hydrocarbons. The methane molecule has resonance bands in the infrared which, to a degree depending on wavelength, restrict the transparent optical depth of the atmosphere. Images at these wavelengths thus probe the atmosphere to particular depths. At Keck, we took narrow band images of Neptune within the 1, 1.6, and $2.1 \mu \mathrm{m}$ infrared bands to compliment the high spatial resolution speckle images. (Speckle imaging through narrow band filters is not possible because this produces much lower signal than through the much wider infrared band filters, plus the fact is, because of methane absorption, the planet is much darker inside the methane bands!)

With the narrow band data, we establish the altitude of the storm features, whether they are in the stratosphere or the troposphere for example, and thus associate them with various haze layers in Neptune's atmosphere. Since the trace hydrocarbons in the atmosphere have such strong light absorption properties, their vertical abundance profiles are very important to understanding the energy transport processes in Neptune's atmosphere. In collaboration with our colleagues at U.C. Berkeley and JPL, we have used an atmospheric modeling program to fit component abundance as a function of altitude to our data.

Io, the innermost moon of Jupiter, is subject to severe tidal stress, which is continually elieved at the surface through volcanic activity. At any given time there are perhaps a ozen volcanoes active, with detectable changes, flare-ups, etc. occurring on time scales of ionths. Lava floes are thought to resurface the moon every 10 years. The lava is generally 
cool at $800^{\circ} \mathrm{C}$ and composed mostly of sulfur, but recent data has indicated that hotter $2000^{\circ} \mathrm{C}$ silicate vulcanism may be occurring in the largest outbursts.

A science spacecraft, Galileo, is presently in orbit around Jupiter, providing a wealth of information about Io as well as the other Galilean moons. Two instruments, the near infrared mapping spectrometer (NIMS) and the solid state imager (SSI) have recorded images of Io volcanoes. However, Galileo was never scheduled to fly very near to Io since the radiation environment at that inner orbit is so severe it would jeopardize the mission. As a result, the NIMS infrared images actually have lower resolution than the Keck diffraction-limited images taken from earth!

We have made repeated observations of Io from Keck, observing Io's volcanoes by their emitted infrared light while in eclipse of Saturn, over the duration of this project. Io subtends only about 0.8 arcseconds as seen from earth, so a high resolution technique like speckle interferometry is necessary to distinguish separate volcanoes on the surface. Using Speckle image reconstruction we have observed new bright, transient outbursts, and variations in the energy of known volcanically active regions. The high spatial resolution allows us to pinpoint the location of the volcanoes and also to bound the extent of the hot regions. Observations in multiple wavelength bands $(2$ and $3 \mu \mathrm{m}$ ) allow us to characterize the temperature of the hot spots, which gives clues to the composition of the lava floes.

In summary, we have demonstrated the enormous potential of diffraction-limited imaging in advance of installation of adaptive optics systems at the world's largest telescopes. We have focused on targets of opportunity which are amenable to the speckle interferometric techniques and also are of current interest to the solar system science community. The repeated measurements at short intervals reveal the "event" nature of the solar system: Io volcanoes flare brightly to high temperature then cool down to near indetectability next to steady glowing caldera, Neptune storms rapidly evolve and encircle the planet in a few days.

In this report, we describe the speckle interferometry technique in more detail in chapter 2 , we present the results from our observations of Titan, Neptune, and Io respectively in chaptcrs 3, 4, and 5, and finally in chapter 6, we discuss the future of high resolution solar system observations from ground based observatories using the new generation of adaptive optics systems. 


\section{IMAGE FORMATION WITH SPECKLE INTERFEROMETRY}

\subsection{Background}

Speckle interferometry has its origin in the early attempts at stellar interferometry. Astronomers realized that by placing a mask over a large aperture telescope with two small holes at the edges of the mask to allow starlight through, interference fringes would appear at the focal plane in a manner similar to Young's two-slit experiment. The fringe spacing (distance betwecn light and dark regions), is inversely proportional to the distance between the holes. In the case of the Michelson stellar interferometer, which was set up on a 6 meter baseline on top of the Hooker 100 inch telescope at Mt. Wilson, this is much smaller than the typical stellar image on photographic plates of the time. Unfortunately the fringes are constantly moving because of air turbulence and can only be viewed by eye, or with a video camera.

Short exposure $(<100 \mathrm{~ms})$ images of starlight, taken without an aperture mask, on a telescope with a diameter of half a meter or more, reveal complex interference patterns (Figure 2.1a), which can be explained as fringes from many pairs of slit holes superimposed. The patterns dance and change with the atmospheric turbulence.

The development of video charge-coupled-device (CCD) technology introduced a detector that was very efficient with the light (approaching $80 \%$ quantum efficiency) and with fast electronic readout capability. Labeyrie (1970) suggested using Fourier transform processing of fast frame rate data images, termed specklegrams, to recover the autocorrelation function of otherwise unresolved astronomical sources. The autocorrelation method has been widely used to make measurements of binary stars (e.g., McAlister et. al. 1984, Henry and McCarthy 1993, Ghez et al 1995, Leinert et al 1993, Koresko et al 1991) and was used to determine the orbit of the moon Charon around the planet Pluto (Beletic et. al. 1989).

Autocorrelation functions are useful for determining simple parametric properties of an object's brightness distribution, however, complete image recovery requires the Fourier phase function as well. Unfortunately, because of the atmospheric turbulence, the phase data of individual specklegrams does not correlate well with the Fourier phase of the source distribution. Knox and Thompson (1974), Weigelt (1977), and Lohmann et al (1983) introduced techniques of determining phase in a more subtle fashion from the data, using higher order moment statistics, notably the "bispectrum" (see the description below). Roddier (1986), recognized the similarity of the bispectrum imaging method to phase closure techniques that had already been used in radio interferometry aperture synthesis for more than a decade to produce very high resolution radio maps of the sky.

Speckle interferometric imaging has been widely used ever since, for example: solar features (e.g. Von Der Luehe 1994), galactic nuclei (e.g., Olivares 1994, Thatte et al 1997), and solar system objects (e.g., Beletic 1992, McCarthy et al 1994, Max et al 1996). 


\subsection{Speckle Imaging Algorithm}

Each specklegram image $I\left(x ; t_{i}\right)$ is related to the object source distribution $O(x)$ by a convolution relationship

$$
I\left(x ; t_{i}\right)=P\left(x ; t_{i}\right) * O(x)
$$

where $x$ is position in the field of view and $P(x)$ is the combined atmosphere and telescope point-spread function. The subscript $i$ indicates the speckle frame number, which is taken at time $l_{i}$. Note that the atmosphere is constantly changing and therefore so is the speckle point spread function. The atmospheric turnover time, that is, the interval during which the point-spread function can be considered approximately constant, depends on the seeing conditions, but is typically in the range of 50 to $100 \mathrm{~ms}$. The time between exposures $\left(t_{i+1}-t_{i}\right)$ depends on the size of the image, speed of the instrument readout electronics, computer storage mechanism, etc. The NIRC camera readout rate with $256 \times 256$ pixel images is approximately once every 2 seconds.

The speckle interferometric image reconstruction algorithm recovers the object by estimating its Fourier phase and Fourier magnitude components in separate steps (see the flow chart of operations in Figure 2). The Fourier magnitude is obtained by applying a standard deconvolution technique to the square root of the average power spectrum. Note that the power spectrum is the Fourier transform of the autocorrelation function, and it is this approach that was first proposed by Labeyrie. The basic equation is

$$
\left\langle\left|\tilde{I}\left(u ; t_{i}\right)\right|^{2}\right\rangle_{i} \approx|\tilde{O}(u)|^{2}|\tilde{P}(u)|^{2}
$$

where $\mathrm{u}$ is the Fourier domain variable (a 2-vector) associated with (the 2-vector) $x$, and $\langle\star\rangle_{i}$ indicates averaging over speckle frames. The power spectrum of the point spread function $|\tilde{P}(u)|^{2}$ is estimated separately using specklegrams of a stellar point source, i.e. of an unresolved star, for which $\tilde{O}(u) \equiv 1$. Actually finding such a source is more difficult to do than one might at first imagine; about half the stars in the sky are binary pairs, and at this resolution, they aren't known! Extra telescope time must be used to collect data on redundant stars so that there is a reasonable chance one of them is a point source.

Deconvolution is, in concept, the operation

$$
|\widehat{\tilde{O}(u)}|=\left\langle\left|\tilde{I}\left(u ; t_{i}\right)\right|^{2}\right\rangle_{i} /|\widehat{\tilde{P}(u)}|^{2}
$$

but is actually performed using one of a variety of clever methods that, for example, guard against numerical problems when the divisor on the right hand side approaches zero.

Fourier phase is recovered through processing the bispectrum of the images. The bispectrum of $I\left(x ; t_{i}\right)$ is defined as

$$
I_{B}\left(u, v ; t_{i}\right) \triangleq \tilde{I}\left(u ; t_{i}\right) \tilde{I}\left(v ; t_{i}\right) \tilde{I}\left(-u-v ; t_{i}\right)
$$


where $\mathrm{u}$ and $\mathrm{v}$ are Fourier domain variables and $I\left(u ; t_{i}\right)$ is the Fourier transform of $I\left(x ; t_{i}\right)$. The bispectrum phase of the object source distribution is, remarkably, equal to the average bispectrum phase of the specklegrams:

$$
\arg \left\{O_{B}(u, v)\right\}=\arg \left\{\left\langle I_{B}\left(u, v ; t_{i}\right)\right\rangle_{i}\right\}
$$

This is because point sources obey the property $\arg P_{B}(u, v ; t) \equiv 0$, which is roughly explained by the fact that coherent phase fronts, although distorted by the path through the atmosphere, have zero integrated phase over all closed triangles in the aperture plane, $\vec{v},(-\vec{u})$, and $(-\vec{u})-\vec{v}$. See Lohmann, et. al. for a complete derivation.

Fourier phase is recovered from bispectrum phase by inverting the recursion relation

$$
\arg \left\{O_{B}(u, v)\right\}=\arg \tilde{O}(u)+\arg \tilde{O}(v)+\arg \tilde{O}(-u-v)
$$

The recursion is started with three assumptions: $\arg \tilde{O}((0,0))=0, \arg \tilde{O}((1,0))=0$, and $\arg \tilde{O}((0,1))=0$. The starting conditions have physical interpretations: 1$)$ wavefront piston is arbitrary so is arbitrarily set to zero, 2) wavefront tip and 3) wavefront tilt only shift the image without changing any other aspect of its structure, and so it can be arbitrarily centered at 0,0 .

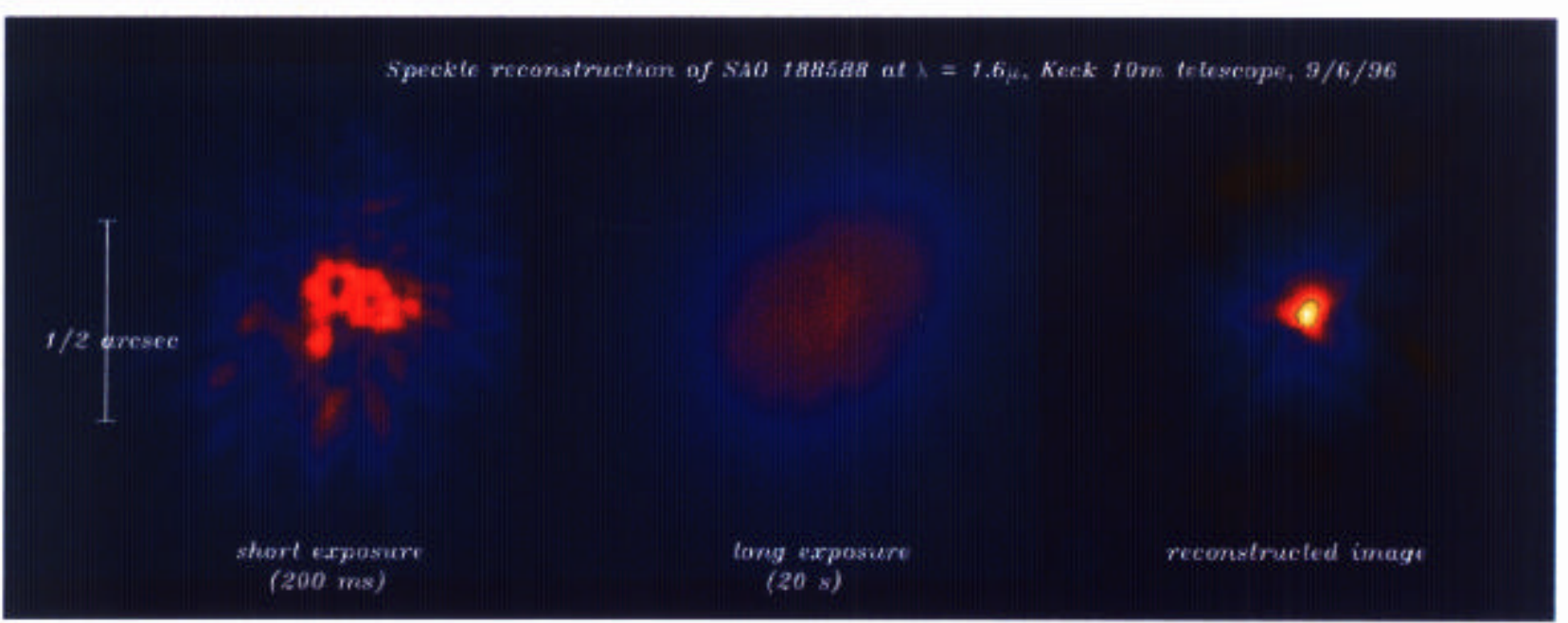

Figure 2.1 - Speckle image formation compared to traditional long exposure image. 


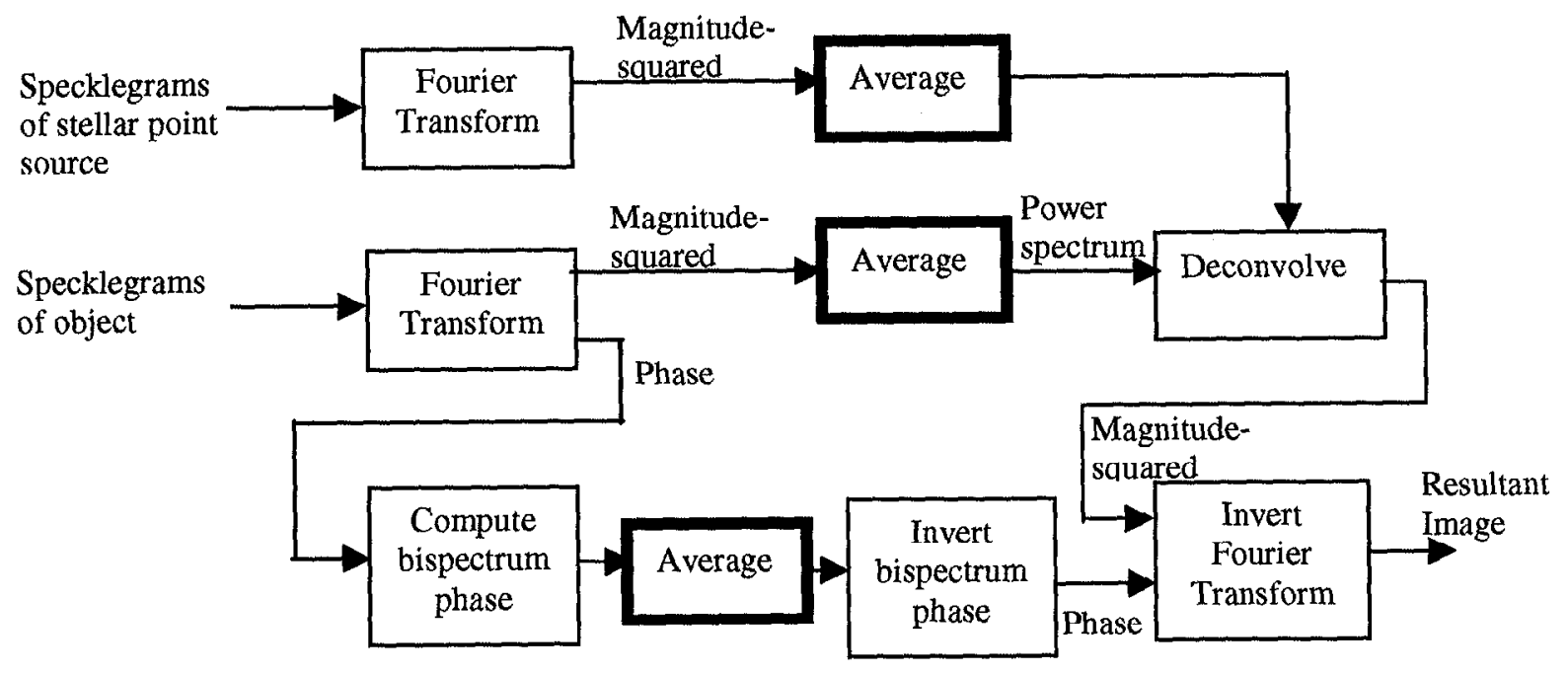

Figure 2.2 - Data flow for speckle image processing. 


\section{BIBLIOGRAPHY FOR CHAPTER 2}

Beletic, J.W., Goody, R.M., Tholen, D.J., 1989. Orbital elements of Charon from speckle interferometry, Icarus, $\mathbf{7 9}$,pp. 38-46.

Beletic, J.W. and R.M. Goody. 1992. Recovery of planetary images by speckle imaging. Applied Optics 31, 6909-6921.

Ghez, A.M., A.J. Weinberger, G. Neugebauer, K. Matthews, and D.W. McCarthy, Jr. 1995. Speckle imaging measurements of the relative tangential velocities of the components of T Tauri binary stars. Astronomical J. 110, 753-765.

Henry, T.J., and D.W. McCarthy. 1993. The mass-luminosity relation for stars of mass 1.0 to 0.08 solar mass. Astronomical I. 106, 773-789.

Knox, K. T. and B.J. Thompson. 1974. Recovery of images from atmospherically degraded short-exposure photographs. Astrophys. J. 193, L45-48.

Koresko, C., S. Beckwith, A. Ghez, K. Matthews, and G. Neugebauer. 1991. An infrared companion to Z Canis Majoris. Astronomical J. 102, 2073-2078.

Labeyrie, A. 1970. Attainment of diffraction limited resolution in large telescopes by Fourier analysing speckle patterns in star images. Astron. Astophys. 6, 85-87.

Lawrence, T.W., D.M. Goodman, E.M. Johansson, and J.P. Fitch. 1992. Speckle imaging of satellites at the Air Force Maui optical station. Applied Optics 31, $6307-6321$.

Lebofsky, L.A., M.A. Feierberg., and A.T. Tokunaga. 1982. Infrared observations of the dark side of Iapetus. Icarus 49, 382-386.

Leinert, C., H. Zinnercker, N. Weitzel, J. Christou, S. T. Ridgway, R. Jameson, M. Haas, and R. Lenzen. 1993. A systematic approach for young binaries in Taurus. Astron. and Astrophys 278, 129-149.

Lohmann, A., G. Weigelt, and B. Wirnitzer. 1983. Speckle masking in astronomy - Triple correlation theory and applications. Applied Optics 22, 4028-4037.

Matthews, K., A.M. Ghez, A.J. Weinberger, and G. Neugebauer. 1996. The first diffraction-limited images from the W.M. Keck Telescope. Publications of the Astronomical Society of the Pacific 108, 615-619.

Max, C.E., D. Gavel, E. Johansson, B. Sherwood, M. Liu and B. Bradford. 1996. Observations of comet $\mathrm{P} /$ Shoemaker-Ievy 9 impact on Jupiter from Lick Observatory using a high resolution speckle imaging camera. IAU Colloquium 156, Baltimore, MD.

McAlister, H.A., W.I. Hartkopf, B.J. Gaston, E.M. Hendry, and F.C. Fekel. 1984. Speckle interferometric measurements of binary stars. IX. Astrophys. J. Suppl. 54, 251-257. 
McCarthy, D.W., J.D. Freeman, and J.D. Drummond. 1994. High resolution images of Vesta at 1.65 microns. Icarus 108 285-297.

Olivares, R.O. 1994. The application of infrared speckle interferometry to the imaging of remote galaxies and AGN, in Astronomical Society of the Pacific, Airborne Astronomy Symposium on the Galactic Ecosystem: from Gas to Stars to Dust, 73, p. 197-198.

Roddier, F. 1986. Triple correlation as a phase closure technique. Optics Communications 60, 145-148.

Von der Luehe, O. 1994. Speckle imaging of solar small scale structure. 2: Study of small scale structure in active regions. Astron. and Astrophys. 281, 889-910.

Weigelt, G.P. 1977. Modified astronomical speckle interferometry 'speckle masking'. Oplics Communications 21, 55-59. 


\section{THE SURFACE AND ATMOSPHERE OF TITAN}

\subsection{Introduction}

Despite decades of observation, Saturn's largest moon Titan remains one of the most mysterious objects in the solar system. Unlike other known planetary satellites, Titan possesses a thick (1.5 bars) atmosphere which, like the Earth's, is composed mainly of nitrogen. It also contains small percentages of methane, hydrogen, and possibly argon. When observed by Voyager I in 1980 at visible wavelengths, only a featureless orange haze could be seen, with no hint of the surface underneath.

Titan's haze layer is produced by photolysis of methane in the upper atmosphere. The photolysis products combine into a complex series of organic molecules and polymers (Yung et al 1984). These organics form a layer that is opaque at visible wavelengths and is partially transparent in the infrared. Calculations indicate that the methane in Titan's atmosphere would be depleted by photolysis on a timescale of $10^{7}$ years (Yung et al 1984), which indicatcs cither that Titan's atmosphere is not in long-term steady-state (Lorenz et al 1997), or that surface-atmosphere interactions replenish the atmospheric methane. It has been suggested that Titan may possess surface or sub-surface reservoirs of liquid hydrocarbons produced by the raining out of methane photolysis products (Lunine et al 1983).

Disk-integrated observations of Titan in the near-infrared (Griffith et al 1991) indicate an atmosphere dominated by methane absorption features. Titan's surface can be observed through narrow windows of the methane spectrum in which light from the surface is not absorbed by the atmosphere. Because haze optical depth is less at infrared wavelengths than in the visible (Rages et al 1983), it is easier to observe photons reflecting from the surface at 1.5-2.5 microns where haze optical depths are generally less than 0.5 , rather than at shorter wavelengths. The maximum contrast in surface features is greatly increased (to $\simeq 30 \%$ ) at near-infrared wavelengths compared to $\simeq 10 \%$ quoted by Smith et al (1996) at the shorter wavelengths observed by the Hubble Space Telescope (HST). The values of contrast that can be obtained depend on both spatial and spectral resolution.

Unresolved, disk-averaged observations of Titan show variations in geometric albedo with time, as well as a north-south brightness asymmetry that is believed to be due to seasonal transport of Titan's haze particles (Hutzell et al 1993,1996). Daily variations are due to a brightness difference between Titan's leading and trailing hemisphere (the leading hemisphere being brighter) as Titan revolves around Saturn with a period of 15.95 days (Lemmon et al 1995). Longer-time variations involve a north/south brightness asymmetry and occur with a period of $\simeq 30$ years, consistent with the orbital period of Titan around the Sun (Lockwood et al 1986). This asymmetry currently has the opposite sign from that observed 18 years ago by Voyager 1 .

Titan has been observed in visible and near-infrared windows by the HST (Smith et al 1996), and in the infrared using adaptive optics technology (Combes et al 1997). These observations have confirmed the brightness asymmetry between Titan's leading and 
trailing hemispheres, as well as the north/south brightness asymmetry. The diffractionlimited resolution achieved by the HST at $0.9 \mu \mathrm{m}$ was $0.09 "(0.045 "$ per pixel), and by adaptive optics $0.14 "$. The deduced surface characteristics indicate a heterogenous surface rather than the global hydrocarbon ocean suggested by loss of methane to the surface at the current rate over the age of the solar system (Yung et al 1984, Lara et al 1994).

Early radar measurements of Titan's albedo (Muhleman et al 1991) suggested a radar reflectivity much too high for liquid hydrocarbons and more consistent with an icy surface. The early values reported by Muhleman et al 1991 were later revised down by a factor of $\sim 2$ (Muhleman et al 1995), due to an error in the calibration procedure (for details see de Pater et al 1995). In contrast to the early reports, the radar reflectivities are therefore not inconsistent with a surface that is covered to some extent by solid or liquid hydrocarbons (Lorenz and Lunine 1997).

It now seems clear, from both observations and theory, that Titan does not possess a global hydrocarbon ocean (Sears 1995, Sagan and Dermott 1982, as well as the infrared and HST data mentioned above). However, it is quite possible that smaller reservoirs of liquid ('seas') exist on Titan but have not been detected by previous observers. Unambiguous detection would require very high resolution measurements to separate the heterogenous areas of the surface as well as high spectral resolution to positively identify the surface components.

In order to help answer such questions, we have used the technique of speckle imaging on the world's largest optical telescope, the W.M Keck 10-meter Telescope, to produce infrared images of Titan at a higher spatial resolution than previous images taken at any wavelength. In addition to producing images of the surface, we have used the data to constrain models of Titan's atmospheric haze layer.

\subsection{Observations and Data Reduction}

Observations were taken on June 27 and September 6, 1996 (UT) using the 10-m W.M. Keck Telescope and the $256 \times 256$ pixel NIRC camera (Matthews et al 1994) in speckle imaging mode (Matthews et al 1996). In this mode a warm image converter provides NIRC with a plate scale of 0.02 arcseconds per pixel. This plate scale gives approximately 40 pixels (20 resolution elements) across the disk of Titan. The K' observations have a spatial resolution of 0.04 arcseconds, which is $\simeq 260 \mathrm{~km}$ on Titan's surface, a higher spatial resolution than any previous observations.

Our obscrvations in Junc were primarily of Titan's trailing hemisphere (Titan orbits synchronously around Saturn, so that its leading and trailing sides are always imaged at greatest eastern and western elongation). 'The central meridian longitudes at the time of observation were 125 degrees for the leading hemisphere and 320 degrees for the trailing hemisphere. The data from September show the leading, brighter hemisphere. Data from June were obtained in the K' band $(1.95 \mu-2.29 \mu)$, and data from September at both K' and $\mathrm{H}(1.49 \mu-1.82 \mu)$. These wavelength ranges include both strong methane absorption 
bands and windows where methane absorption is nearly zero, so that light can penetrate to the surface.

Although using custom narrow-band filters arranged around the methane absorption bands, as Combes et al (1997) do, would provide somewhat higher contrast on surface features, speckle imaging requires a high signal-to-noise in each individual frame, so the broad-band filters must be used. The contrast in our images remains very high, and the presence in the images of a significant atmosphere-only component actually allows more precise determination of atmospheric parameters (see section 4).

The observations taken are summarized in Table 1. Each data set consists of 100 images, with a 0.200 second exposure time per image. Each such stack typically takes 90 seconds to complete. Each frame also has 8 (June) or 200 (September) dark/bias exposures associated with it, which are used to calibrate drifts in the DC bias level of NIRC's electronics. The number of dark/bias exposures was increased in September because the time-varying bias and read noise in the dark frames were dominant noise sources in the June data.

Before the speckle analysis procedure began the data were pre-processed as conventional infrared images. The instrument dark current and (time-varying) bias were removed using bias frame stacks associated with each data set. Data were sky-subtracted using 300-frame blank-sky observations taken shortly before or after the Titan and reference images. A median filtering technique was then applied to each image to remove residual columnar patterns caused by drifts in the instrument electronics. The resulting images were flatfielded using flats constructed from twilight sky images.

Figure 1 shows the images obtained from speckle reconstruction of the data. Figure 1a is the leading hemisphere at $\mathrm{K}^{\prime}$, reconstructed from 5 sets of 100 speckle frames that were shifted to a common center and averaged to produce the image. Figure 1b shows the leading hemisphere at $\mathrm{H}$ band, reconstructed from an average of 4 sets of 100 speckle frames, and Figure 1c shows the trailing hemisphere at K', reconstructed from 2 sets of 400 speckle frames. Near the center of the satellite, infrared photons pass through only a thin layer of reflective haze, and are either absorbed by the methane below the haze or reach the surface. On the other hand, near the limb the pathlength through the high-altitude haze is longer and photons have a greater chance of being scattered or reflected before they reach the absorbing methane layer. This, combined with a greater amount of haze in the southern hemisphere, leads to a strong southern limb brightening. The observed limb brightening is used to constrain models of the atmosphere.

\subsection{Speckle Image Reconstruction}

Titan is difficult to analyze from the ground because of its small angular size, about 0.8 arcsecond in diameter. This is roughly the size of the point spread blurring caused by turbulence in the Earth's atmospherc. Conscquently, most ground-based observations prior to 1996 measured only properties of the integrated disk.

Since then, high resolution techniques have been developed that can to a certain degree 
overcome the atmosphere and reach a resolution limit set by the diffractive properties of the telescope optics. The diffraction limited resolution varies directly with telescope diameter, so the larger (e.g. Keck 10 meter) telescopes on the ground can in theory obtain higher resolution than HST, with its 2.4 meter aperture in space.

In our work, we applied the Speckle interferometric imaging technique described in Chapter 2. Several hundred short exposure images of Titan are taken over the course of each observing night, along with several hundred short exposure images of a star to use as a point spread function calibration source. These data are processed in groups of 100 frames (see below) with the speckle reconstruction software as described in Chapter 2 to form the high resolution images.

Since the Keck Telescope has an altitude-azimuth mount, the angle between the infrared detector's $x$ axis and the north direction on the sky (the "parallactic angle") depends on the position of the object in the sky and will change as the telescope tracks. For ordinary long exposure observations this effect is removed by an image rotator built into the Cassegrain tower holding the NIRC infrared camera. In speckle mode, however, this rotator is turned off (locked in position with respect to the camera). This is done primarily to simplify calibration of the resulting observations, since the structure of the point spread function (PSF) depends on the optics of the camera, the complex hexagonal shape of the Keck primary mirror, and small phasing errors between different segments of the mirror, with the latter two being the dominant terms. By disabling the rotator we can ensure that both the telescope and camera components of the PSF are precisely the same for our Titan observations and for our observations of PSF reference stars. Of course, this means that Titan as seen by the camera will appear to rotate with time. This can be dealt with in several ways. One approach is to rotate the Fourier transforms of the image data on a frame-by-frame basis (Matthews et al 1996). We have adopted the simpler approach of processing each set of 100 Titan frames with no rotation, since each frame spans a sufficiently short time that the rotation is negligible (ranging from 0.1 to 0.7 degree over the course of a hundred frame set, with the larger number corresponding to half a pixel at the edge of Titan's limb). Each final speckle-processed image was then rotated so that Titan north was in the $+y$ direction. This has the advantage of averaging out most speckle artifacts, which have a fixed orientation relative to the camera; true Titan features can be recognized by their (apparent) rotation from frame set to frame set.

The reconstructed images clearly show a diffraction-limited spatial resolution of 0.04 arcseconds (because of the pixel size of 0.02 arcseconds, the resolution at $\mathrm{H}$ band is limited by Nyquist sampling to 0.04 arcseconds rather than the theoretical limit of 0.03 arcseconds). Further cross-checking of the reconstruction, along with an indication of the level of image artifacts, is accomplished by applying the algorithm to test stars not used in the deconvolution step. We found that the technique left residual artifacts at about the $5 \%$ level of contrast. The implication is that measurements of brightness, and indirectly, surface reflectance, in dark regions are trustworthy to an upper bound of $5 \%$ of the brightness of the bright regions.

We measured an apparant magnitude for Titan of $8.18 \pm .1$ at 2.1 microns and $8.04 \pm .1$ 
at 1.6 microns. This gives a geometric albedo of 0.057 at 2.1 microns and 0.07 at 1.6 microns. This is consistent with measurements made by previous observers (Griffith et al 1991).

\subsection{Atmosphere Model}

In order to separate the contributions of light due to atmospheric scattering and light reflected from the surface, we used a radiative transfer model that simulates the absorption, reflection and scattering of photons from Titan's atmosphere and a constant-albedo surface (Toon et al 1989). This model has previously been applied to Titan by Toon et al (1992), and Hutzell et al $(1993,1996)$. The model uses a computationally-efficient algorithm to solve the two-stream approximation in an inhomogeneous multiple-scattering atmosphere. The Eddington method is used to determine the two-stream coefficients. The accuracy of this method is usually better than $10 \%$ for the calculation of radiative streams, except for large solar zenith angles where the error may reach 10-20\%. Since other sources of error (e.g. the values of the scattering coefficients determined from fractal particle models) are not known to better than 10-20\%, this provides sufficient accuracy for our purposes.

We assume that the atmosphere does not vary in longitude and varies smoothly with latitude as described below. We fit the data as well as possible to such an atmosphere, in particular near the limb of Titan, where the intensity is dominated by atmospheric scattering and is essentially independent of the surface albedo. Any residual that remains when we remove this atmosphere from the data is assumed to be due to surface features. The atmosphere is divided into 3 vertical layers, each of which has a value for the haze and gas optical depths. The haze is confined to the top two layers, with methane underneath. Models with more layers (up to 10) were also tried, with results similar to the 3-layer model. The methane is assumed to be completely absorbing within the fraction of the filter that is in the methane band (this fraction is $61 \%$ for $\mathrm{K}$ ' and $69 \%$ for $\mathrm{H}$ ), and to be transparent outside of the methane absorption band. Haze single scattering albedos and forward/backward scatter asymmetry factors at $\mathrm{H}$ and $\mathrm{K}^{\prime}$ band (given in Table 2) are derived from a fractal model of Titan's haze particles (Rannou et al 1995). In order to account for the observed north/south haze asymmetry, the haze optical depth was assumed to decrease linearly from the southern limb to the equator, and from the equator to the northern limb using independent slopes for each hemisphere.

Our standard model assumes that the surface of Titan reflects as a Lambert disk; this is a surface for which the reflected intensity varies as the cosine of the angle from the disk center. This is only one extreme in the possible continuum of reflectance models. The opposite extreme is a more lunar-like pattern in which the reflectance does not vary across the disk. We have also constructed models with a constant reflectance (which will be referred to as "lunar-like") to test the sensilivity of the models to this unknown parameter. As might be expected, since the lunar-like surface reflects more efficiently at high zenith angles, the effect of using a lunar-like model is to produce a somewhat lower surface reflectance at the edges of the disk; the central values are essentially unchanged. The best-fit values of the haze optical depth for the lunar-like models are slightly less at 
the north and south poles (e.g. 0.26 vs. 0.3 at the south pole and 0.06 vs. 0.1 at the north pole for the $\mathrm{K}^{\prime}$ band). In theory it would be possible to use these models to determine whether Titan's surface is indeed more "Lambert-like" or "lunar-like"; however, we do not find enough difference between the results of the two models to make this distinction.

The modeling process produces a theoretical image of Titan's atmosphere with effectively infinite spatial resolution, To compare this to our observations, the model must be degraded to the true resolution of our images, by convolving it with the point spread function (PSF) of our data. In the case of perfect speckle data, with infinite signal-tonoise and no systematic calibration errors, the point spread function would simply be the inverse of the modulation transfer function (MTF) of the telescope - an Airy disk for a round telescope, a somewhat more complex shape for the hexagonal Keck primary mirror. In practice, noise in the data (which becomes significant at high spatial frequencies in the power spectrum) and calibration errors (due to changes in the atmospheric turbulence properties between the images of Titan and the images of the PSF reference star) will degrade the resolution of the images.

We estimated this degradation at K'-band by speckle-processing images of one star SAO 128735 - in the same fashion as our Titan data, using a different star as a reference. (See Table 1). (A similar PSF, constructed from observations of 188588, was used for the H-band data.) The resulting PSF was then used to convolve our models. Inspection of the resulting image, particularly the brightness profile of the image in the area beyond Titan's limb (which will depend primarily on the PSF, not the model), indicated that the actual Titan data was sharper than the SAO 128735 data. This is most likely due to fluctuations in the atmospheric properties ("seeing") over the course of the observations; if the SAO 128735 observations were taken in a time of slightly worse seeing than the SAO 128780 observations used for the speckle processing, the resulting image will be slightly blurred. Conversly, convolving the model with the theoretical "perfect" PSF produced an image that was clearly sharper still than our actual observations. We thererfore developed a mixed PSF produced by a weighted average of the star/star PSF and the theoretical PSF. We evaluated the goodness of fit by comparing the brightness profile beyond the limb of the convolved model with our observations; since the model naturally has no flux beyond its limb, the shape of the convolved image in this region is dependent only on the PSF used. All these deviations from the theoretical PSF are relatively small - the images all achieve the 0.04 " diffraction limit, with the variations being in the amount of light present in a low-level halo surrounding the central core. A $50 \%$ theoretical $/ 50 \%$ observed PSF weighting was found to produce results that most closely correspond to our observed data. The effect of varying the PSF on the total haze opacity is relatively small; the best-fit model with the theoretical PSF has a total haze opacity in the southern hemisphere of 0.26 , compared with 0.3 using the composite PSF. The effect on the surface reflectance was small; an increase in reflectance of 0-0.02 across the disk, with the largest effect near the limb. The reflectance of the dark regions remained below 0.05 .

Once the model was corrected for the point spread function of the data, there were two main steps to the fitting process. First, the atmospheric parameters - particularly the optical depth in the scattering haze - were determined by fitting a model with uniform 
surface albedo to the limb of our observations. Second, those haze parameters were used to recover a surface reflectance map by removing the contribution to the light due to the atmosphere, and by correcting for absorption and scattering of surface light.

In the first step, a series of models with varying haze optical depths and surface albedos was generated. The albedo was assumed to be uniform across Titan's surface. This is clearly not the case. However, since the "goodness of fit" of each model was evaluated. primarily in the limb regions, this means we are merely assuming that the albedo in the southern and northern polar regions is relatively uniform. In both our observations and other groups' studies of Titan (Smith et al 1996, Combes et al 1997) the southern and northern polar regions appear devoid of bright features. In addition, the assumed albedo couples only weakly into our atmospheric model for two reasons. First, the limb emission is clearly dominated by scattered light from haze, particularly in the south, as can be seen from the sharp limb brightening; the relative contribution due to haze is further magnified since our observations span a wavelength range that only partially probes the surface. Second, due to the absorption and scatlering of light reflected from the surface on the way up, the overall brightness at the limb is only a weak function of the assumed surface albedo.

The model parameters - haze optical depth as a function of latitude, and (uniform) surface albedo - were adjusted to produce the model that best fit our observations for several longitudinal slices five pixels wide near the northern and southern limbs (Fig. 2 a-c). We consider our recovered optical depths to be good to $\pm 20 \%$ : a model with $20 \%$ less haze clearly undersubtracts the limb brightening while a model with $20 \%$ more haze clearly oversubtracts (Figure 2d and Figure 7).

Figure 2 shows plots of brightness as a function of image row for three vertical (N/S) slices through our image. It can be seen that the model reasonably fits the data near the limbs. Away from the limbs, deviations from the model are presumably due to non-uniform surface albedo; in particular, the region where the data are well below the model in figure $2 c$ indicates a surface reflectance that is well below the uniform 0.05 reflectance used in this model.

Once the best-fit haze parameters were determined, the model was used to generate an "atmosphere-only" map with an assumed surface albedo of zero (Figure 3). This atmosphere-only map was then separated from our data lo produce an image of the residual emission, due to the (non-uniform, non-zero) surface. The map was then corrected for atmospheric absorption and scattering, and angle-of-incidence effects (assumed to be Lambertian) by comparing it to a model with a constant surface albedo. In modelling our data for the trailing hemisphere at $K$ ' band, which has lower signal-to-noise than the leading hemisphere data, we have used the atmosphere parameters which best fit the leading hemisphere. Along the with leading hemisphere models at $\mathrm{H}$ and $\mathrm{K}$ ' bands, this gives the surface reflectance maps shown in Fig. 6. We note that, according to our model, $28 \%$ of the light from the planet comes from the surface (on a disk-averaged basis) at $\mathrm{H}$ band, and $30 \%$ at $\mathrm{K}$. The remainder of the light is reflected from the haze. 


\subsubsection{Results: Atmosphere}

Table 2 shows the deduced haze optical depths in the $\mathrm{K}^{\prime}$ and $\mathrm{H}$ bands. These results are not in complete agreement with either of two previous globally averaged models (Figure 4). The fractal haze particle model of Rannou et al (1995) has about twice the global average optical depth obtained from our analysis while the earlier spherical particle model (McKay et al 1989) gives a value about four times greater. Our results also indicate that Titan's haze optical depth currently (as of 1996) varies by a factor of $\simeq 3$ from south to north.

Both the spherical and the fractal haze models shown in Figure 4 are adjusted to fit the geometric albedo of Titan in spectral range from 0.3 to 0.9 microns. The opacity of the atmosphere at longer wavelengths is an extrapolation from this fit. For spherical particles more mass of haze is needed to fit the visible than for the fractal model because the optical cross section of the fractal particles is larger per unit haze mass than for spherical particles. However at longer wavelengths where the absorption and scattering are in the Rayleigh regime, the optical cross section is more nearly proportional to the total haze mass and is not sensitive to the particle shape. Thus the fractal optical depth falls below the spherical optical depth in the infrared.

Our data indicate that the optical depths in the infrared are somewhat lower than predicted by the fractal model. This could indicate that the fractal model is still not an adequate representation of Titan's haze either because the fractal dimension used $(D=2)$ is incorrect or the basic assumption of radial symmetry of the fractal particles is not valid. Many condensates do form string-like aggregates that could have significantly different optical properties than the fractal models of Rannou et al 1995. Also, a portion of the difference may be due to seasonal variations between our observations and the Rannou et al data. We note that our haze optical depth values at $2 \mu \mathrm{m}$ (particularly in the northern hemisphere of Titan) are in good agreement with the results of Griffith et al (1991), who found a disk-integrated haze Mie scattering optical depth at $2 \mu \mathrm{m}$ of 0.1-0.13.

Knowledge of Titan's haze optical depth at infrared wavelengths is useful for comparing and refining models of the structure of Titan haze particles (Rannou et al 1995, McKay et al 1989), which can then be used to extrapolate haze optical depths to other wavelengths (see Figure 4). The Cassini mission to Saturn, launched in October 1997 and scheduled for arrival in 2004, carries the Imaging Science Subsystem (ISS), a CCD imaging system with the potential of acquiring images of Titan's surface with spatial resolutions of tens of meters. The ISS carries a 0.94 micron filter tuned to the methane window expressly for imaging the surface and a polarizer to help block singly scattered photons, but the proportion of nonscattered photons reflected from the surface is a steep function of the haze optical depth. Multiply-scattered photons have the potential to severely degrade the spatial resolution of the ISS, possibly blurring features to as much as several kilometers. The $\mathrm{H}$ and $\mathrm{K}^{\prime}$ haze optical depths determined from our Keck speckle observations, combined with future shorter wavelength optical depth measurements, can be used to refine performance constraints for the Cassini ISS. 
Our results indicate lower optical depths than predicted by either the fractal or spherical particle models, good news for the Cassini mission. These lower optical depths imply that the surface can be easily imaged in the $1 \mu \mathrm{m}$ methane window, albeit with the caveat that there could be low-level haze which is not included as a separate parameter in our model.

\subsubsection{Results: Surface}

Titan's surface albedo provides clues to its surface composition. Suggested surface compositions range from liquid hydrocarbon oceans (Lunine 1983) to solid rock or ice / hydrocarbon mixtures (Muhleman et al 1991, Griffith et al 1991). Figure 5 shows the surface reflectance of Titan's leading hemisphere at $\mathrm{K}$ ' band in a false-color map. Figure 6 shows surface reflectance for both leading and trailing hemispheres at $K$ ' and for the leading hemisphere at H. Titan's surface is clearly heterogenous, with bright areas of reflectance $>0.15$ in the $H$ band, and very dark areas with reflectance $<0.05$ at both $\mathrm{H}$ and $\mathrm{K}^{\prime}$. The surface images have an average reflectance for the September image (45 degrees away from the usual "bright side" reported in the literature) of $0.059 \pm 0.027$ at 2.0 microns, with an equivalent geometric albedo of $0.046 \pm 0.018$. This is higher than $2 / 3$ of the average reflectance since the bright feature is relatively central. At 1.6 microns the average reflectance is $0.085 \pm 0.04$ and the equivalent geometric albedo $0.066 \pm 0.027$. In June-centered on the dark side of Titan-the average 2.0 micron reflectance is $0.027 \pm 0.027$ and the geometric albedo is $0.023 \pm 0.018$. If we neglect the outermost five-pixel annulus of each map (which is most sensitive to errors in the atmosphere fitting, but also favors the brighter terrain) we get average reflectances of $0.079 \pm 0.023,0.111 \pm 0.034$, and $0.038 \pm 0.023$ for September 2.0 microns, September 1.6 microns, and June 2.0 microns respectively.

These results can be compared to disk-integrated infrared albedos from previous observers, which are summarized in Lorenz and Lunine (1997). The previous data show a wide range of albedo values both for Titan's bright (leading) and dark (trailing) hemispheres. For example, values given for the geometric albedo of the bright hemisphere at $1.6 \mu$ range from 0.07 (Griffith et al 1991), to 0.4 (Lemmon et al 1995). Past spectroscopic studies of Titan's surface have provided differing results primarily because of photometric errors associated with narrow slit spectroscopy. At this time it is difficult to say which values are most accurate, although our values appear to be in reasonable agreement with Griffith et al (1991).

Previous observations have not detected the regions we find on the leading hemisphere with infrared reflectance $<0.05$, presumably because these regions have a modest spatial extent and have been blurred by scattered light from the brighter regions at lower spatial resolution. To test our conclusion that these regions are very dark, we have constructed atmospheric models with a $20 \%$ lower haze optical depth (Figure 7a). These models, which have a clearly undersubtracted southern limb, still have a reflectance in the dark region of $\leq 0.05$. Models with a $20 \%$ higher haze optical depth produce dark regions with reflectance $<0$ (Figure $7 \mathrm{c}$ ). We therefore are confident that these regions are very dark at both $\mathrm{H}$ and $\mathrm{K}^{\prime}$ band. These regions could be larger than our map indicates, covering most of the area to the left of Figure 5 (west in Titan coordinates) if we have either underestimated 
the haze optical depths in the north, or if the PSF scatters significant light from the bright feature.

\subsection{Discussion and Interpretation of Surface Features}

The reflectances we have obtained here for 'I'itan's surface may be compared to those of other solar system bodies and laboratory materials in order to narrow down the range of candidate materials. Table 3 lists the geometric albedos of some solar system bodies and of other Titan surface candidate materials at 1.6 and 2.1 microns.

From a comparison of Figures 6(a) and 6(b) it can be seen that the bright region on the leading hemisphere is somewhat brighter at $H$ than at $K$ ', which suggests that it may be composed of water ice (which is dark at 2 microns and somewhat brighter at 1.6), or an ice-rock mixture. The bright feature has a similar spatial extent to the bright region seen by other observers (Smith et al 1996, Combes et al 1997), and we find, in agreement with previous observations (Combes et al 1997), that the bright region has 'peaks' with reflectance $10-15 \%$ greater than the surrounding bright regions. The general shape of the bright region, including the westward extension near the north pole in Figure 5, is quite similar to that seen by the HST at a wavelength of $0.94 \mu \mathrm{m}$ (Smith et al 1996).

The trailing hemisphere of Titan at the $\mathrm{K}^{\prime}$ band has an overall brightness less than the leading hemisphere, and we again find (Figure 6c) that the albedo is not uniform. The trailing hemisphere shows features qualitatively similar to those observed by Combes et al (1997), namely, that there is a somewhat brighter region in the northern hemisphere (reflectance $\simeq 0.07$ ), and a less bright feature (reflectance $\simeq 0.05$ ) in the southern hemisphere.

The reflectance of the very dark regions is too low for water ice such as is seen, for example, on Jupiter's moon Europa, although very smooth ice which would appear dark in the infrared remains a possibility. Another possibility, that the dark material could consist of deposits similar to those found on the other Saturnian satellites Iapetus and Hyperion, is unlikely since the Iapetus dark material is too bright in the infrared (Vilas et al 1996).

Saturn's satellite Phoebe, on the other hand, is quite dark in the infrared. Phoebe is believed to be a captured asteroid because of its spectral similarity to C-type asteroids (their infrared albedo is similar; see Table 3). However, even Phoebe-like material is not as dark as the darkest regions we observe on Titan's leading hemisphere. Another possibility is that the dark material is similar to laboratory-produced "tholins" (Khare et al 1984), perhaps mixed with water ice. However, the reflectance of "tholins" is in the range of 0.06-0.08, probably too high to explain the very low reflectance of the dark regions on the lower left quadrant of Figure 5.

In theory, these patches of material could also have an extra-Titan source, such as asteroidal dust. However, because of Titan's thick atmosphere, any dust falling in from outside the atmosphere would be spread rather evenly over Titan's surface. We cannot 
envision a scenario where much material would naturally accumulate in restricted patches on the surface.

Finally, we note that the localized areas of dark material could be the elusive "lakes" or "seas" of liquid hydrocarbon that have long been predicted to exist on Titan's surface. We find that the reflectance of these regions is sufficiently low at 1.6 and 2.1 microns that the presence of liquid hydrocarbons cannot be ruled out. If photochemical models (Lara et al 1994) are correct, the amount of liquid found in these regions is probably not enough to keep the atmosphere resupplied with methane.

In view of the many possibilities for Titan's surface composition, it is useful to discuss what the distribution of bright and dark regions evident at high resolution can tell us. The dark material on Titan's leading hemisphere appears to be restricted to confined areas. Compared to features on the Earth, the size of the dark area seen in the lower left of Figure 5 is about as big as Hudson's Bay, and somewhat larger than the Black Sea. The dark areas may be large basins (impact or volcanic basins) that are covered by dark material which comes from the interior of the satellite or is brought in by a dark impactor (which would probably be vaporized in the process). Since Titan is a differentiated object, it is hard to see how material that appears to bc most similar to primitive asteroids could be indigenously present on its surface, particularly only in restricted areas. If liquid hydrocarbons exist on Titan's surface, the bright and dark patterns could be due to the redistribution of liquid to low-lying areas. Or, the surface of Titan could be continually covered by dark material precipitating out from the atmosphere which is washed from only the higher-lying (bright) areas by methane rain, exposing bright ice underneath (Griffith et al 1991).

From what we know of Titan's atmosphere it seems likely that Titan's surface is continually impacted by material from above, whether in solid or liquid form. The fate of this material, whether washed to low-lying areas by methane rain, collected in underground reservoirs, or accumulating as liquid on the surface, remains uncertain.

\subsection{Conclusions}

Observations of Titan using speckle imaging at the W.M. Keck 10-m telescope have achieved a higher contrast and spatial resolution than previous images. They clearly show a variety of surface features on Titan, and yield detailed information about the optical properties of Titan's atmosphere. Atmospheric modeling indicates haze optical depths at $\mathrm{H}$ and $\mathrm{K}$ ' that are smaller than predicted by spherical or fractal particle models. The northsouth asymmetry in atmospheric brightness suggests a large difference in haze density in the two hemispheres which would be expected with seasonally-dependent global circulation (Hourdin et al 1995). Variations in surface reflectance, on scales that can only be resolved by high-resolution imaging, indicate a highly heterogenous surface, including bright areas consistent with ice or rock-ice, and dark areas with reflectance $<0.05$ which may be covered with organic solids or liquid hydrocarbons. 
Table 3.10bservations of Titan

\begin{tabular}{|c|c|c|c|c|c|}
\hline Data Set & Object & Filter & Time $(\text { UTC })^{1}$ & $\begin{array}{c}\text { Parallactic } \\
\text { Angle }\end{array}$ & $\begin{array}{l}\text { Reference } \\
\text { Star Set }(s)^{2}\end{array}$ \\
\hline \multicolumn{6}{|l|}{ June 27, 1996} \\
\hline 709 & Titan & $\mathrm{K}^{\prime}$ & $14: 25: 12$ & $\begin{array}{r}+55.37 \\
\end{array}$ & $749,758,767$ \\
\hline 718 & Titan & $\mathrm{K}^{\prime}$ & $14: 27: 46$ & +54.85 & $749,758,767$ \\
\hline 727 & Titan & $\mathrm{K}^{\prime}$ & $14: 30: 03$ & +54.38 & $749,758,767$ \\
\hline 736 & Titan & $\mathrm{K}^{\prime}$ & $14: 32: 32$ & +53.85 & $749,758,767$ \\
\hline 749 & SAO 109256 & $\mathrm{~K}^{\prime}$ & $14: 37: 26$ & +54.33 & $n / a$ \\
\hline 758 & SAO 109256 & $\mathrm{~K}^{\prime}$ & $14: 39: 59$ & +53.76 & $\mathrm{n} / \mathrm{a}$ \\
\hline 767 & SAO 109256 & $\mathrm{~K}^{\prime}$ & $14: 42: 23$ & +53.21 & $\mathrm{n} / \mathrm{a}$ \\
\hline 825 & Titan & $\mathrm{K}^{\prime}$ & $15: 04: 59$ & +45.08 & $749,758,767$ \\
\hline 834 & Titan & $\mathrm{K}^{\prime}$ & $15: 07: 31$ & +44.23 & $749,758,767$ \\
\hline 843 & Titan & $\mathbf{K}^{\prime}$ & $15: 10: 05$ & +43.33 & $749,758,767$ \\
\hline 852 & Titan & $\mathbf{K}^{\prime}$ & $15: 12: 47$ & $\begin{array}{r}+42.35 \\
\end{array}$ & $749,758,767$ \\
\hline \multicolumn{6}{|c|}{ September 6, 1996} \\
\hline 458 & Titan & $\mathrm{K}^{\prime}$ & $11: 31: 19$ & +7.92 & 490 \\
\hline 473 & Titan & $\mathbf{K}^{\prime}$ & $11: 46: 38$ & -2.57 & 490 \\
\hline 476 & Titan & $\mathbf{K}^{\prime}$ & $11: 51: 55$ & -6.18 & 490 \\
\hline 483 & Titan & $\mathrm{K}^{\prime}$ & $11: 58: 00$ & -10.29 & 490 \\
\hline 490 & SAO 128780 & $\mathrm{~K}^{\prime}$ & $12: 05: 10$ & -11.73 & $n / a$ \\
\hline 517 & SAO 128735 & $\mathrm{~K}^{\prime}$ & $12: 44: 05$ & -35.97 & $\mathrm{n} / \mathrm{a}$ \\
\hline 524 & Titan & $\mathrm{K}^{\prime}$ & $12: 50: 45$ & -38.81 & 517 \\
\hline 604 & SAO 128762 & $\mathrm{H}$ & $14: 44: 05$ & -62.18 & $\mathrm{n} / \mathrm{a}$ \\
\hline 614 & Titan & $\mathrm{H}$ & $14: 56: 42$ & -64.19 & 604 \\
\hline 617 & Titan & $\mathrm{H}$ & $15: 01: 48$ & -64.62 & 604 \\
\hline 620 & Titan & $\mathrm{H}$ & $15: 06: 40$ & -65.01 & 604 \\
\hline 623 & Titan & $\mathrm{H}$ & $15: 11: 33$ & -65.38 & 604 \\
\hline
\end{tabular}

1. UTC time from instrument control computer, which contains an unknown offset of several seconds relative to true UTC

2. Data set used for speckle calibration (see section 3) 
Values for scattering parameters and best-fit haze optical depths at 1.6 and $2.1 \mu \mathrm{m}$. Values for $\mathrm{g}$ and $\omega$ were taken from the fractal haze particle model of Rannou et al (1995). Haze optical depth is assumed to decrease linearly from the southern limb to the equator and from the equator to the northern limb.

\begin{tabular}{|c|c|c|c|c|c|}
\hline Wavelength & $\mathrm{g}$ & $\omega$ & $\tau_{\mathrm{S}}$ & $\tau_{\mathrm{E}}$ & $\tau_{\mathrm{N}}$ \\
\hline $1.6 \mu \mathrm{m}$ & 0.40 & 0.97 & 0.52 & 0.26 & 0.16 \\
\hline $2.1 \mu \mathrm{m}$ & 0.20 & 0.85 & 0.30 & 0.15 & 0.10 \\
\hline
\end{tabular}

$\mathrm{g}=$ scattering asymmetry factor

$\omega=$ single-scattering albedo

$\tau_{\mathrm{S}}=$ haze optical depth at southern limb

$\tau_{\mathrm{E}}=$ haze optical depth at equator

$\tau_{\mathrm{N}}=$ haze optical depth at northern limb 
Table 3.3

Albedos of Candidate Materials

\begin{tabular}{lcc}
\hline Object & $1.6 \mu m$ albedo & $2.1 \mu m$ albedo \\
\hline Europa $^{\mathrm{a}}$ (ice) & 0.25 & 0.11 \\
Moon $^{\mathrm{b}}$ (rock) & 0.3 & 0.3 \\
Iapetus (dark regions) $^{\mathrm{c}}$ & 0.11 & 0.10 \\
Phoebe $^{\mathrm{d}}$ & 0.06 & 0.05 \\
C-type asteroids $^{\mathrm{e}}$ & $0.04-0.06$ & $0.04-0.06$ \\
Titan tholinı $^{\mathrm{f}}$ & $0.06-0.08^{*}$ & $0.06-0.08^{*}$ \\
liquid hydrocarbons $^{\mathrm{g}}$ & $<0.02^{*}$ & $<0.02^{*}$ \\
Titan dark areas (this work) & $<0.05^{*}$ & $<0.05^{*}$ \\
Titan bright areas (this work) & $0.12-0.18^{*}$ & $0.10-0.13^{*}$
\end{tabular}

*These numbers are normal reflectance values; all others are geometric albedos ${ }^{a}$ Clark and McCord 1980

${ }^{b}$ Lorenz 1997

${ }^{c}$ Lebofsky et al 1982

${ }^{d}$ Degewij et al 1980, scaled to values from Tholen and Zellner 1983

eTholen and Barucci 1989

$f_{\text {Khare et al }} 1984$

${ }^{g}$ Khare et al 1990 


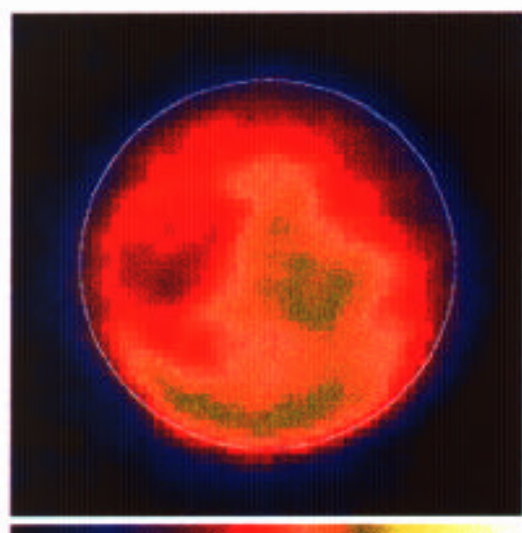

Figure 1a

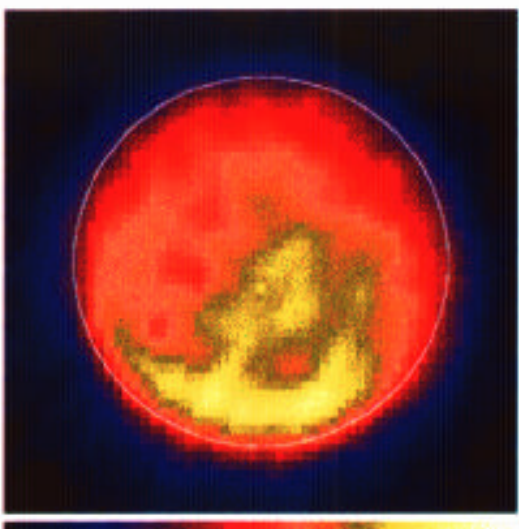

Figure 1b

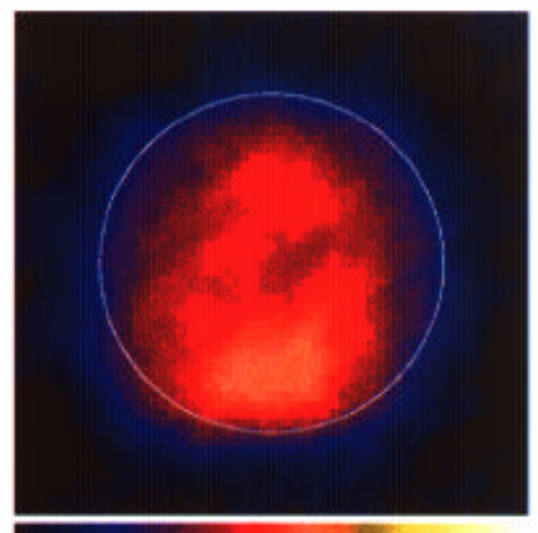

Figure 1c

Figure 3.1: Speckle processed images of Titan. Figure 1a shows the leading hemisphere at $\mathrm{K}^{\prime}$ (2.1 microns); Figure $1 \mathrm{~b}$ is the leading hemisphere at $\mathrm{H}$ (1.6 microns), and Figure 1c is the trailing hemisphere at K'. The central longitude for the leading hemisphere is $125^{\circ}$; and for the trailing hemisphere $320^{\circ}$. Titan north is up and west is to the left. The mapping of intensity to color is the same for $1 \mathrm{a}$ and $1 \mathrm{c} ; 1 \mathrm{~b}$ has a different color mapping since Titan's integrated intensity is greater in the $\mathrm{H}$ band. Units of scale are flux in $10^{-18} \mathrm{Watts} / \mathrm{m}^{2} / \mu /$ pixel 


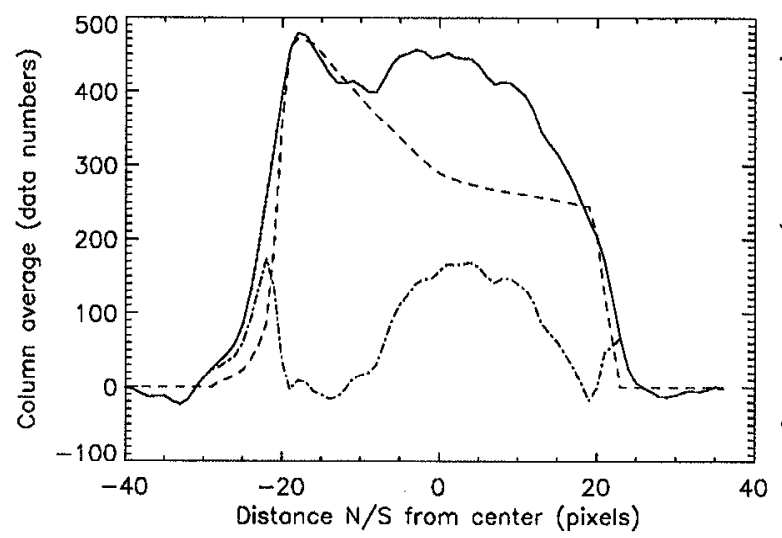

A

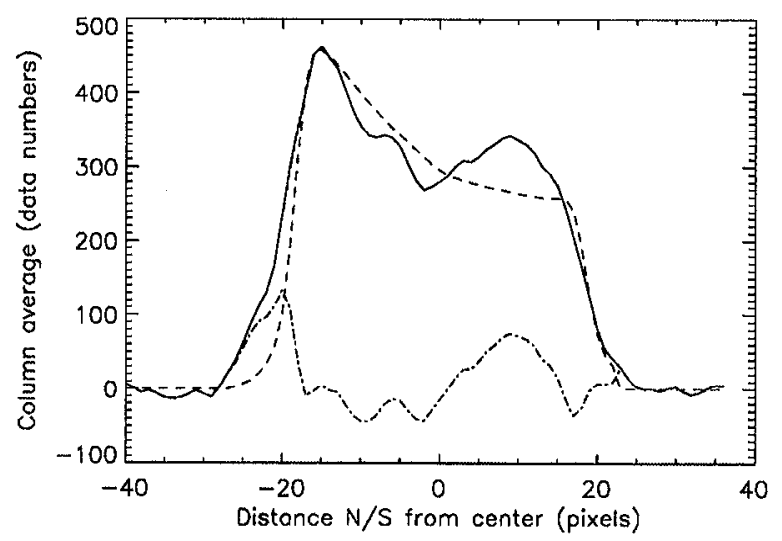

C

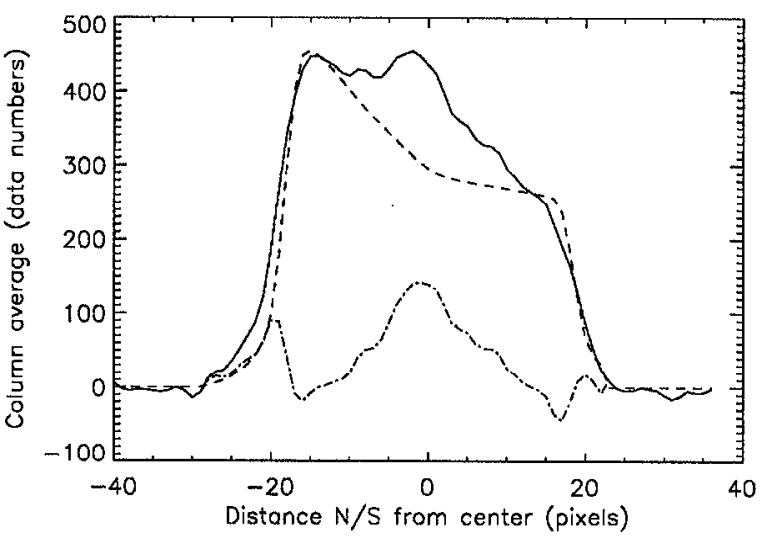

$\mathrm{B}$

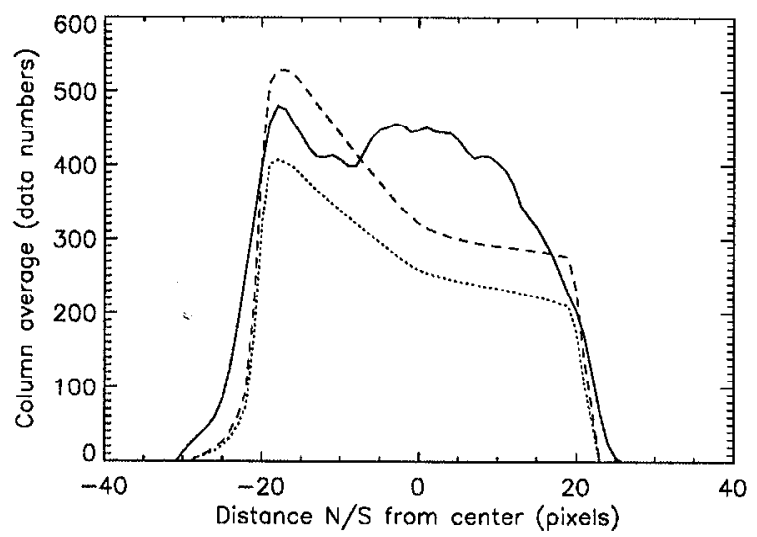

$\mathrm{D}$

Figure 3.2: Fitting the model to the data. Figures 2a-c show N/S slices (average of 5 pixels) through the satellite at three different longitudes compared to the average of 5 pixels of the model atmosphere plus a surface with a constant albedo of 0.05 . The solid line is data, the dotted line is the model, and the dot-dash line is the residual (difference between data and model). Data are fit to the northern and southern limbs (hatched areas). The positive residual near the center of the image is due to a bright surface feature; the negative residual in figure $2 c$ (just north of the southern limb) is one of the dark regions. Figure 2d shows a center N/S slice of the data (solid line), an atmosphere model with $20 \%$ less haze than the nominal value (dotted line), and $20 \%$ greater haze (dashed line). These models are clearly less adequate fits to the northern and southern limbs than the nominal model shown in Figure 2 a-c. The surface reflectance produced by the $20 \%$ greater and $20 \%$ less haze models is shown in Figure 7. 


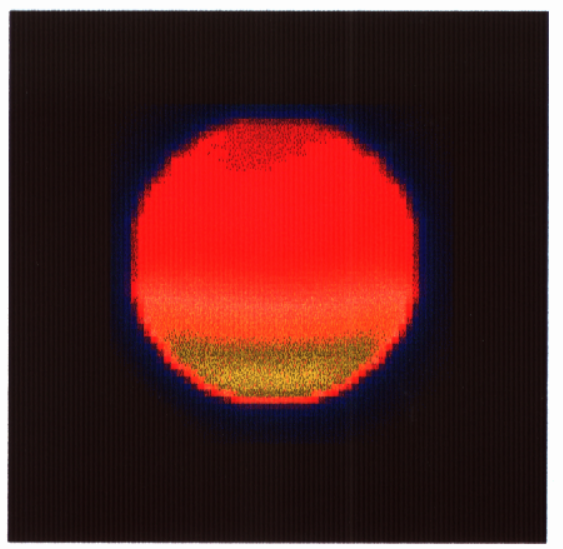

Figure 3.3: Brightness of a Titan model atmosphere at $K^{\prime}$ band shown in grayscale map. Units of scale are flux in $10^{-18}$ Watts $/ \mathrm{m}^{2} / \mu /$ pixel (same units as Figure 1). This image is for a surface albedo of zero, so that all light comes from the atmosphere. The model for $\mathrm{H}$ band is very similar. Strong southern limb brightening is due to higher haze abundance in the southern hemisphere.

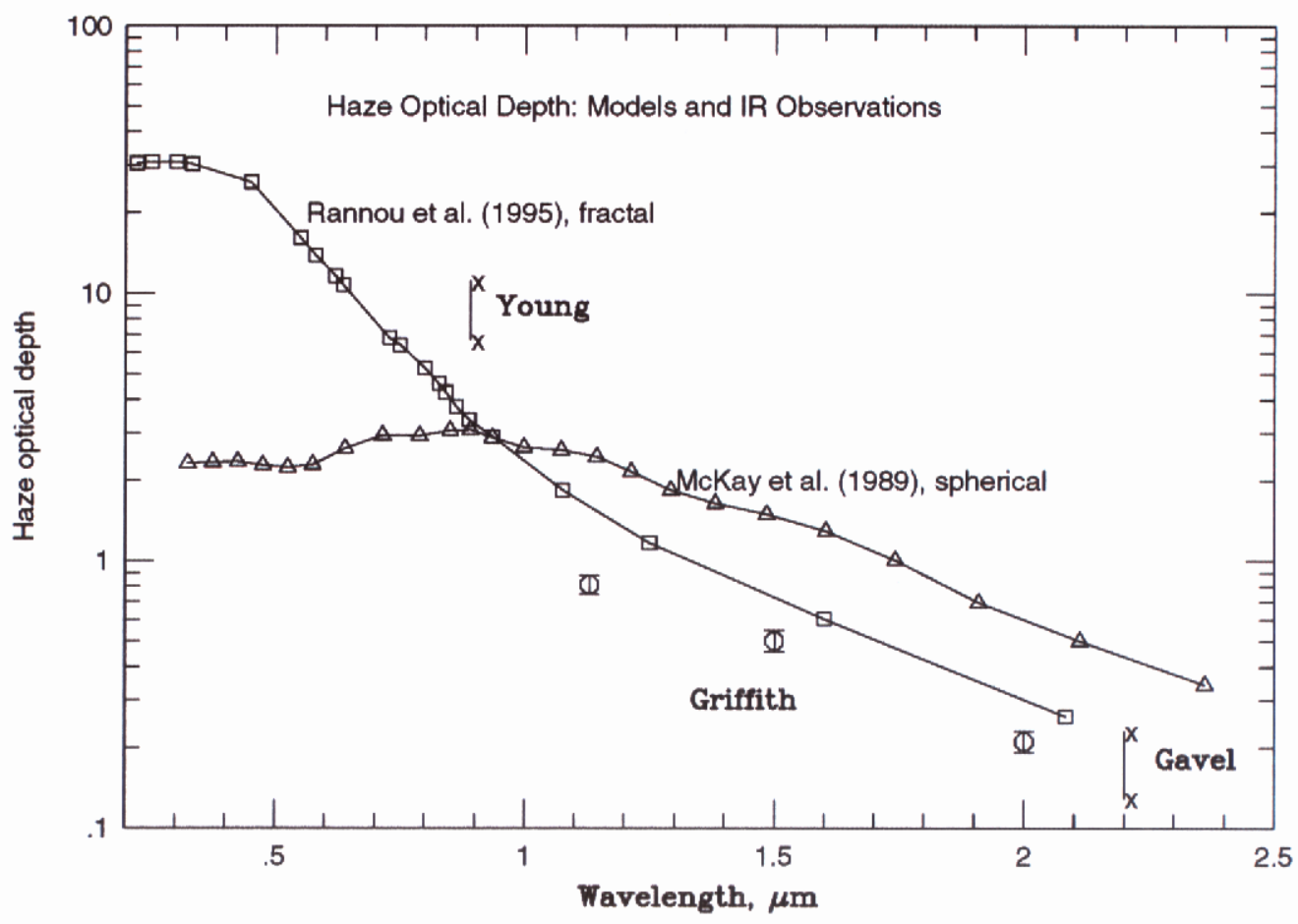

Figure 3.4: The optical depth of Titan's haze layer at various wavelengths. Two models are shown: the Mckay et al (1989) spherical particle model, and the Rannou et al (1995) fractal particle model. Data are shown for H and K' band; numbers are given for the value found at the northern and southern limbs along with $20 \%$ error bars. 


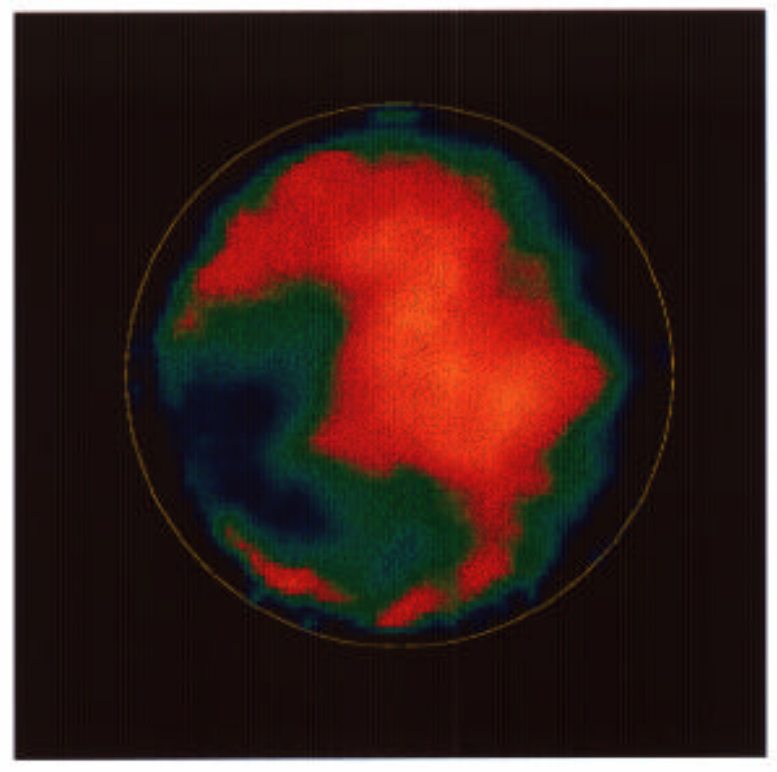

Figure 3.5: False-color map of Titan's surface reflectance in the K' band, showing the leading hemisphere (central longitude $125^{\circ}$ ).
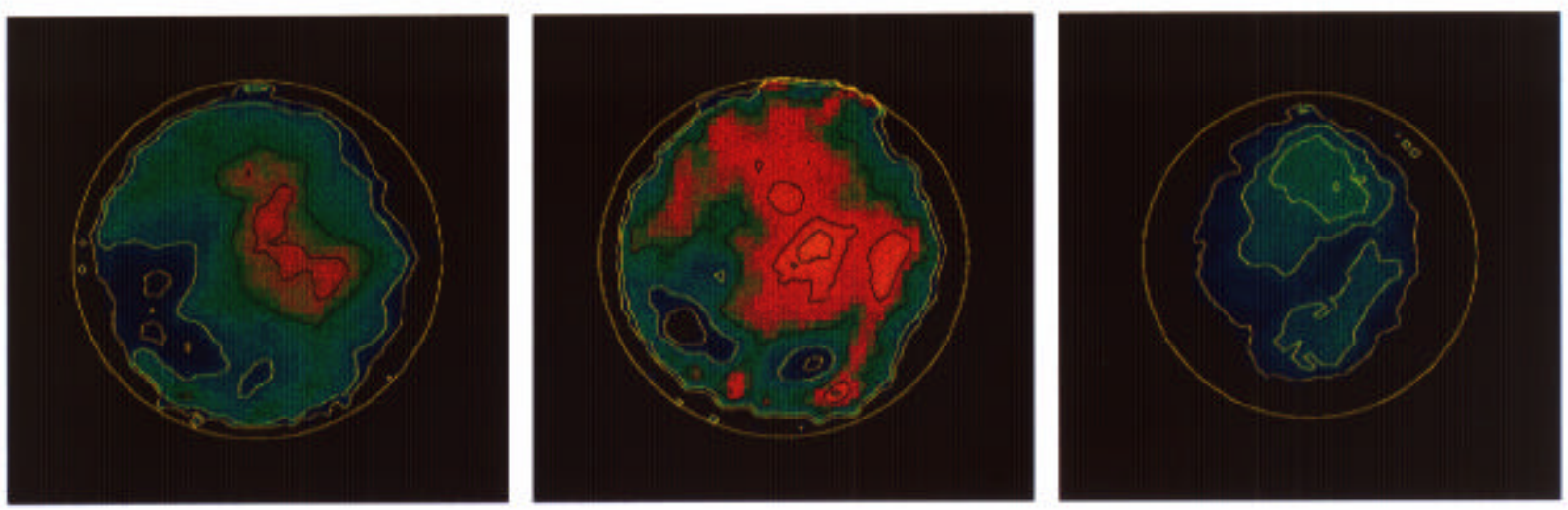

Figure 3.6: Maps of Titan's surface reflectance. Figure 3.6a is the leading hemisphere at K'; Figure 3.6b shows the leading hemisphere at H; and Figure 3.6c shows the trailing hemisphere at $\mathrm{K}$ '. 

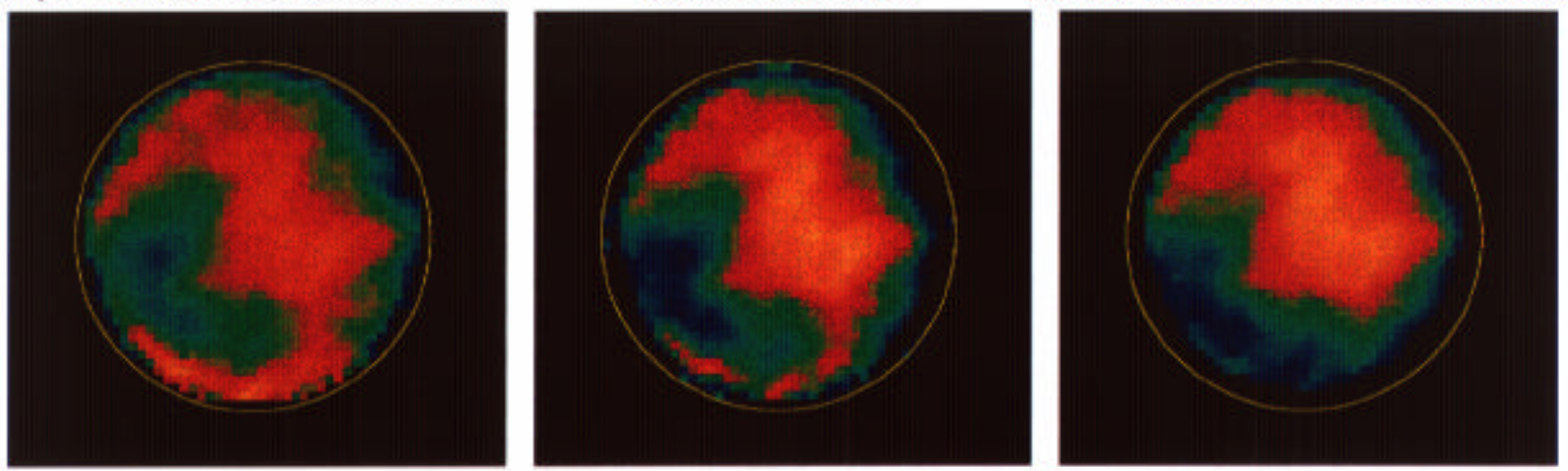

Figure 3.7: Recovered surface reflectance for models with $20 \%$ less haze optical depth than the nominal value (3.7a), $20 \%$ more haze optical depth (3.7c), and the nominal value (3.7b, nominal values are given in Table 1$)$. The model with $20 \%$ less haze shows clear undersubtraction of the southern limb, but the darkest reflectances are still $<0.05$. The model with $20 \%$ more haze produces surface reflectance in the dark regions that is $<0$, which is clearly unphysical. 


\section{BIBLIOGRAPHY FOR CHAPTER 3}

Beletic, J.W. and R.M. Goody. 1992. Recovery of planetary images by speckle imaging. Applied Optics 31, 6909-6921.

Clark, R.N., and T.B. McCord. 1980. The Galilean satellites: new near-infrared reflectance measurements (0.65-2.5 microns) and a 0.325-5 micron summary. Icarus 41, 323-339.

Combes, M., A. Coustenis, L. Vapillon, E. Gendron, R. Wittemberg, and R. Sirdey. 1996. Images of Titan's surface in the Near-IR with ADONIS. Bull. Amer. Astron. Soc., DPS meeting \#28, \#20.04.

Combes, M. L. Vapillon, E. Gendron, A. Coustenis, O. Lai, R. Wittemberg, and R. Sirdey. 1997. Spatially resolved images of Titan by means of adaptive optics. Icarus 129, 482-497.

Degewij, J., D.P. Cruikshank, and W.K. Hartmann. 1980. Ncar-infrared colorimetry of J6 Himalia and S9 Phoebe: A summary of $0.3-2.2 \mu \mathrm{m}$ reflectances. Icarus 44, 541-547.

de Pater, I., P. Palmer, D.L. Mitchell, S.J. Ostro, D.K. Yeomans, and L.E. Snyder. 1994. Radar aperture synthesis observations of asteroids. Icarus 111, 489-502.

Ghez, A.M., A.J. Weinberger, G. Neugebauer, K. Matthews, and D.W. McCarthy, Jr. 1995. Speckle imaging measurements of the relative tangential velocities of the components of T Tauri binary stars. Astronomical J. 110, 753-765.

Griffith, C.A., T. Owen, and R. Wagener. 1991. Titan's surface and troposphere, investigated with ground-based near-infrared observations. Icarus 93, 362-378.

Henry, T.J., and D.W. McCarthy. 1993. The mass-luminosity relation for stars of mass 1.0 to 0.08 solar mass. AJ 106, 773-789.

Hourdin, F., Talagrand, O., Sadourny, R., Courtin, R., Gautier, D., and C.P. McKay. 1995. Numerical simulation of the general circulation of the atmosphere of Titan. Icarus 117, 358-374.

Hutzell, W.T., C.P. McKay, and O.B. Toon. 1993. Effects of time-varying haze production on Titan's geometric albedo. Icarus 105, 162-174.

Hutzell, W.T., C.P. McKay, O.B. Toon, and F. Hourdin. 1996. Simulations of Titan's brightness by a two-dimensional haze model. Icarus 119, 112-129.

Khare, B.N., W.R. Thompson, C. Sagan, E.T. Arakawa, and J.J. Lawn. 1990. Optical constants of solid ethane from 0.4 to $2.5 \mu \mathrm{m}$. Bull. Am. Astron. Soc. 22, 1033.

Khare, B.N., C. Sagan, E.T. Arakawa, F. Suits, T.A. Callcott, and M.W. Williams. 1984. Optical constants of organic tholins produced in a simulated Titanian atmosphere: from soft X-ray to microwave frequencies. Icarus 60, 127-137. 
Knox, K. T. and B.J. Thompson. 1974. Recovery of images from atmospherically degraded short-exposure photographs. Astrophys. J. 193, L45-48.

Koresko, C., S. Beckwith, A. Ghez, K. Matthews, and G. Neugebauer. 1991. An infrared companion to Z Canis Majoris. AJ 102, 2073-2078.

Labeyrie, A. 1970. Attainment of diffraction limited resolution in large telescopes by Fourier analysing speckle patterns in star images. Astron. Astophys. 6, 85-87.

Lara, L.M., R.D. Lorenz, and R. Rodrigo. 1994. Liquids and solids on the surface of Titan: results of a new photochemical model. Planet. Space Sci. 41, 5-14.

Lawrence, T.W., D.M. Goodman, E.M. Johansson, and J.P. Fitch. 1992. Speckle imaging of satellites at the Air Force Maui optical station. Applied Optics 31, $6307-6321$.

Lebofsky, L.A., M.A. Feierberg., and A.T. Tokunaga. 1982. Infrared observations of the dark side of Iapetus. Icarus 49, 382-386.

Leinert, C., H. Zinnercker, N. Weitzel, J. Christou, S. T. Ridgway, R. Jameson, M. Haas, and R. Lcnzen. 1993. A systematic approach for young binarics in Taurus. Astron. and Astrophys 278, 129-149.

Lemmon, M.T., E. Karkoschka, and M. Tomasko. 1995. Titan's rotation: surface feature observed. Icarus 113, 27-38.

Lockwood, G.W., B.L. Lutz, D.T. Thompson, and S.E. Bus. 1986. The albedo of Titan. Astrophys. J. 303, 511-520.

Lohmann, A., G. Weigelt, and B. Wirnitzer. 1983. Speckle masking in astronomy - Triplc correlation theory and applications. Applicd Optics 22, 4028-4037.

Lorenz, R.D., and J.I. Lunine. 1997. Titan's surface reviewed: the nature of the bright and dark terrain. Planet. Space Sci. 45, 981-992.

Lorenz, R.D., C.P. McKay, and J.I. Lunine. 1997. Photochemically driven collapse of Titan's atmosphere. Science 275, 642-644.

Lunine, J.I. D.J. Stevenson and Y.L. Yung. 1983. Ethane ocean on Titan. Science 222, 1229-1230.

Matthews, K., A.M. Ghez, A.J. Weinberger, and G. Neugebauer. 1996. The first diffraction-limited images from the W.M. Keck Telescope. Publications of the Astronomical Society of the Pacific 108, 615-619.

Matthews, K., and B.T. Soifer. 1994. Infrared Astronomy with Arrays: the Next Generation. I. McLean, ed. (Dordrecht: Kluwer Academic Publishers), p. 239.

Max, C.E., D. Gavel, E. Johansson, B. Sherwood, M. Liu and B. Bradford. 1996. Observations of comet $\mathrm{P} /$ Shoemaker-Levy 9 impact on Jupiter from Lick Observatory using a high resolution speckle imaging camera. Presented at IAU Colloquium 156, Baltimore, MD May 9-12, 1995, available as a Technical Re- 
port, Lawrence Livermore National Lab, Livermore CA, United States.

McAlister, H.A., W.I. Hartkopf, B.J. Gaston, E.M. Hendry, and F.C. Fekel. 1984. Speckle interferometric measurements of binary stars. IX. Astrophys. J. Suppl. 54, 251-257.

McCarthy, D.W., J.D. Freeman, and J.D. Drummond. 1994. High resolution images of Vesta at 1.65 microns. Icarus 108 285-297.

McKay, C.P., J.B. Pollack, and R. Courtin. 1989. The thermal structure of Titan's atmosphere. Icarus 80, 23-53.

Muhleman, D.O., A.W. Grossman, and B.J. Butler. 1995. Radar investigations of Mars, Mercury, and Titan. Annual Review of Earth and Planetary Science 23, $337-374$.

Muhleman, D.O., A.W. Grossman, B.J. Butler, and M.A. Slade. 1991. Radar reflectivity of Titan. Science 248, 975-980.

Olivares, R.O. 1994. The application of infrared speckle interferometry to the imaging of remote galaxies and AGN, in Astronomical Society of the Pacific, Airborne Astronomy Symposium on the Galactic Ecosystem: from Gas to Stars to Dust, v. 73 , p. $197-198$.

Rages, K., J. B. Pollack, and P.H. Smith. 1983. Journal of Geophysical Research 88, p. $8721-8728$.

Rannou, P., M. Cabane, E. Chassefiere, R. Botet, C.P. McKay, and R. Courtin. 1995. Titan's geometric albedo: role of the fractal structure of the aerosols. Icarus 118, 355-372.

Roddier, F. 1986. Triple correlation as a phase closure technique. Optics Communications 60, 145-148.

Sagan, C., and S.F. Dermott. 1982. The tides in the seas of Titan. Nature 300, 731-733.

Sears, W.D. 1995. Tidal dissipation on Titan. Icarus 113, 39-56.

Smith, P.H., M.T. Lemmon, R.D. Lorenz, L.A. Sormovksy, J.J. Caldwell and M.D. Allison. 1996. Titan's surface, revealed by HST imaging. Icarus 119, 336-349.

Thatte, N., A. Quirrenbach, R. Genzel, R. Maiolino, and M. Tecza. 1997. The Nuclear Stellar Core, the Hot Dust Source, and the Location of the Nucleus of NGC 1068. AJ 490, 238-246.

Tholen, D.J. and M.A. Barucci. 1989. Asteroid Taxonomy, in Asteroids II, R.P. Binzel, T. Gehrels and M.S. Matthews, eds. University of Arizona Press, Tucson., 298-315.

Toon, O.B., C.P. McKay, T.P. Ackerman, and K. Santhanam. 1989. Rapid calculation of radiative heating rates and photodissociation rates in inhomogenous 
multiple scattering atmospheres. J.G.R. 94, 16287-16301.

Toon, O.B., C.P. McKay, C.A. Griffith, and R.P. Turco. 1992. A physical model of Titan's aerosols. Icarus 95, 24-53.

Vilas, F., S.M. Larson, K.R. Stockstill, and M.J. Gaffey. 1996. Unraveling the zebra: clues to the Iapetus dark material composition. Icarus 124, 262-267.

Von der Luehe, O. 1994. Speckle imaging of solar small scale structure. 2: Study of small scale structure in active regions. Astron. and Astrophys. 281, 889-910.

Weigelt, G.P. 1977. Modified astronomical speckle interferometry 'speckle masking'. Optics Communications 21, 55-59.

Yung, Y.L., M. Allen, and J.P. Pinto. 1984. Photochemistry of the atmosphere of Titan: comparison between model and observations. Astrophys. J. (Suppl. Ser.) 55, 465-506. 


\section{NEPTUNE'S ENERGY BALANCE AND DYNAMIC WEATHER PATTERNS}

\subsection{Introduction}

The energy output of Neptune exceeds that of solar insolation by a factor of 2.5 , which is much more than is measured on any of the other giant planets (Pearl and Conrath 1991). Therefore, Neptune is expected to be dynamically very active, as was indeed confirmed by the images taken with Voyager 2 . These images, taken at visible wavelengths, show prominent dark and bright spots on the planet, as well as some bright wispy cloud features (Smith et al. 1989). Over the years much time variability in Neptune's spots has been reported, with time scales varying from hours to years (Ingersoll et al. 1995, Baines et al. 1995). The most striking observation has been that the Great Dark Spot and its bright companion cloud, the most prominent features in Voyager and ground based images during many years, had disappeared when Neptune was reimaged by HST in 1991 (Sromovsky et al. 1995) and 1995 (Hammel et al. 1996).

Resolving Neptune with groundbased imaging is difficult, since the planet's angular size is only 2.35 arcseconds at mean opposition. The Great Dark Spot and its bright companion show up as a large diffuse source in conventional images; resolving this feature and distinguishing smaller features on the disk requires use of the HST (e.g., Sromovsky et al. 1995, Hammel et al. 1996), adaptive optics (Roddier et al. 1997, 1998), or speckle imaging (Gibbard et al. 1998). In this chapter we present diffraction limited speckle images of Neptune at nearinfrared wavelengths taken with the $10 \mathrm{~m}$ W.M. Keck telescope. Our resolution is 0.04 arcseconds, corresponding to a scale of about $500 \mathrm{~km}$ on Neptune, which is similar to the best IIST observations at visible (5000 $\AA$ ) wavelengths.

Observations of Neptune at visible wavelengths probe cloud layers in the planet's troposphere (at pressure $\mathrm{P} \gtrsim 0.1$ bars), whereas observations at infrared wavelengths, where methane gas is the main source of opacity, are sensitive to features above the main cloud deck, in Neptune's stratosphere. Transient infraredbright features have been noticed on Neptune for years (Hammel 1989, Lockwood et al. 1991, Hammel et al. 1992). One such feature accompanied the Great Dark Spot imaged by Voyager 2 (Smith et al. 1989). It is not known, however, if bright stratospheric features are typically associated with disturbances in the troposphere such as the GDS.

\subsection{Observations and Data Reduction}

We observed Neptune with the $10 \mathrm{~m}$ W. M. Keck telescope (The W. M. Keck telescope is jointly owned and operated by the University of California and the California Institute of Technology) on Mauna Kea, Hawaii, on September 61996 (UT) and October $10-13$ 1997 (UT). During this time Neptune's subsolar latitude was $-25^{\circ}$, so that our images show primarily Neptune's southern hemisphere. Table 4.1 summarizes dates, times, and wavelengths of the observations.

We observed Neptune both in speckle imaging mode (see Chapter 2), and using con- 
ventional observing techniques (we refer to these as narrowband images). For both types of observations we used the facility's near-infrared camera (NIRC, Matthews and Soifer 1994), which is equipped with a $256 \times 256$ pixel Santa Barbara Research Corporation InSb array. The pixel size is 0.151 arcseconds, corrsponding to $3400 \mathrm{~km}$ at Neptune. For speckle observations a plate scale changer is inserted into the light path to produce a scale of 0.02 arcseconds per pixel, wellmatched to the telescope's 2 micron diffraction limit $(0.04$ arcseconds) and yielding a resolution of $500 \mathrm{~km}$ at Neptune. The conventional images were taken at several different wavelengths between 1 and $3 \mu \mathrm{m}$ in order to probe different altitudes in Neptune's stratosphere. The filters used were h210 (2.113-2.134 $\mu \mathrm{m}), \mathrm{ch}_{4}$ $(2.190-2.345 \mu \mathrm{m})$, feii $(1.638-1.656 \mu \mathrm{m})$, and oii $(1.231-1.241 \mu \mathrm{m})$. Table 4.1 shows the list of narrowband images and reference stars for each night. Typical angular resolution for these observations is 0.6 arcseconds.

The narrowband (low resolution) data were processed by correcting for bias and saturation, removing bad pixels, flatfielding, and sky subtracting.

In the Speckle interferometric processing, several hundred short exposure frames of Neptune, along with a corresponding number of frames of a point spread function calibrator star, were taken on each night of observing. Images were typically speckle processed in stacks of 100 exposures to avoid the paralactic angle smearing described in Chapter 3 , Section 3. Each individual frame was inspected and edited before the images were speckle processed to form a high resolution image. Each of the result images from 100-frame speckle processing was then rotated so that sky north is up. The final image of Neptune for the several hundred frame group was formed by averaging these rotated 100-frame result images. The rapid rotation of Neptune about its polar axis causes the final image to be blurred in longitude by approximately $4^{\circ}$ on the planet ( 3 pixels or $2.5 \%$ of the total Neptune disk size of 118 pixels) during the $\sim 10$ minute time interval between the first and last image frames of a typical 300 frame group.

Before the speckle imaging procedure was applied, the data was preprocessed in the same manner as conventional infrared images. The instrument dark current and (time varying) bias were removed using bias frame stacks associated with each data set. Data were sky subtracted using 300 frame blank sky observations taken shortly before or after the Neptune and reference star images. A median filtering technique was then applied to each image to remove residual columnar patlerns caused by drifts in the instrument electronics. The resulting images were flat fielded using flats constructed from twilight sky images.

\subsection{Modeling Neptune's Stratosphere}

By observing Neptune in narrow bands at a scale of 0.15 arcseconds per pixel (the resolution of these nonspeckle images is dependent on the seeing, typically around 0.6 arcseconds, see Table 4.1), we are able to get information about the vertical structure of Neptune's cloud features. These narrow bands probe different altitudes in Neptune's atmosphere. The shortest wavelength filter, oii, probes down to the troposphere. The feii 
filter, which is the closest filter to the broad $\mathrm{H}$ band used for speckle imaging, and the $\mathrm{h} 210^{-}$ filter sample the lower stratosphere (around 0.1 to 0.01 bars), and the $\mathrm{ch}_{4}$ filter samples the upper stratosphere around $10^{-3}$ bars.

In order to compare the narrowband images, we have calculated the I/F (ratio of reflected intensity to solar flux) for each image. For September 61996 the data were calibrated using the photometric standard star FS34. For October 13 the standard photometric star SJ9182 was used to calibrate the intensity of the data. Solar flux values were taken from Arvessen et al. (1969).

Figures $4.1-4.5$ show images obtained on the five nights of observation. Figure 1 shows the speckle $\mathrm{H}$ band image and the three narrowband images obtained on September 6 1996, and Figures 4.2 - 4.5 show speckle and narrowband images from October 10-13 1997. Actual pixels are shown, without interpolation.

We have used a model atmosphere program from Baines and Hammel (1994) to model the low resolution multiwavelength images from Neptune. Since we observed in different methane absorption bands to probe several altitudes in Neptune's stratosphere, it is crucial to have an accurate model to represent the absorption as a function of wavelength. The program was therefore updated to yield the most accurate representation of the methane absorption bands, through the use of k-distribution coefficients. Two sets of coefficients have been published (Baines et al. 1993: wavelength range $1.60-2.52 \mu \mathrm{m}$; Irwin et al. 1996: wavelength range $1.05-5.0 \mu \mathrm{m}$ ), where we have used and compared the two sets where they overlap. As we will show, the differences in the coefficients lead to substantial differences in the modeled brightness.

A schematic of the atmospheric structure and haze layers relevant to our modeling is summarized in Fig. 4.6a. We adopted a $\mathrm{CH}_{4}$ fractional abundance of $3 \%$ in the troposphere (Baines and Smith, 1990), which followed the saturated vapor curve at higher altitudes. The photochemistry predicts the formation of hydrocarbons as $\mathrm{C}_{2} \mathrm{H}_{2}, \mathrm{C}_{2} \mathrm{H}_{6}, \mathrm{C}_{4} \mathrm{H}_{2}, \mathrm{HCN}$, $\mathrm{C}_{3} \mathrm{H}_{8}, \mathrm{C}_{3} \mathrm{H}_{6}$ (e.g. Romani and Atreya 1989; Romani et al. 1993), decreasing the $\mathrm{CH}_{4}$ abundance in the stratosphere below the saturated vapor curve. We adopted a mixing ratio of 0.00035 (constant with altitude) as the nominal value, but kept this as a free parameter. Condensation of the hydrocarbons leads to the production of haze layers in Neptune's stratosphere, as sketched in Fig. 4.6a. As in Baines and Hammel (1994), we preserved the ratio in number density between the various haze layers to equal the acetylene/ethane and diacetylene/ethane mixing ratios from Romani et al. 1993. We left the total haze column density as a free parameter, and similarly the altitude of the haze layers.

We chose to model the lowresolution data of October 13; on this day we obtained the highest resolution ( 0.6 arcsecond) images, on which we could identify a (hopefully) spotfree region on Neptune's disk. Scans through these spotfree regions on the planet at the four wavelengths are shown in Fig. 4.6b. The dotted lines point at the lowest intensities on each scan, which represent the most spotfree region on the planet. As can be gathered from the images, it is very hard to identify a spotfree region, so one must keep in mind that the intensities are upper rather than lower limits. The progression from a very dim 
planet in the ch $_{4}$ band to a bright object in the oii band is very dramatic; intensities differby nearly a factor of 20 .

Observations in the oii band probe down to the troposphere, and are sensitive to both the total stratospheric and the tropospheric methane haze layers. To match Neptune's brightness in the oii filter, the number density of the methane haze layer needs to be increased by a factor of 3 above that adopted by Baines and Hammel (1994). The model results are relatively insensitive to the precise altitude of the stratospheric haze. In the other three filters one probes Neptune's stratosphere, and stratospheric parameters become dominant. The stratospheric methane abundance is a critical parameter in modeling Neptune's brightness. In addition, there are profound differences in model results using the kdistribution coefficients from Baines et al. (1993) versus those by Irwin et al. (1996), as exemplified in Fig. 4.7.

\subsection{Spot Rotation Periods}

In the October 1997 speckle data we see the same bright spots on different days, and hence we were able to calculate rotation periods for these features. The bright feature at $-45^{\circ}$ was observed on October 10 and 11 (this feature is just coming onto the limb in the image from October 12); on October 11 and 12 there is a feature at $-30^{\circ}$. In order to find rotation periods, the image from each date was projected onto a latitude-longitude grid, and the leading edge, center, and trailing edge of the spots was identified. Then a rotation period was calculated from the movement in longitude of the spots over the observation interval ( $\simeq 23$ hours). The rotation period was taken as the average of the movement of the leading edge, center, and trailing edge (since these spots may change shape over time, it is difficult to know which measurement is the most accurate). Two sources of error identified were in locating the center of the disk of Neptune and in pinpointing the edges of the bright features. We estimate this error as \pm 3 pixels per image, which gives a root mean squarc error of $8.5^{\circ}$ for the difference in spot location from night to night.

The rotation periods we find are $17.1 \pm 0.3$ hours for the spot at $-45^{\circ}$ and $17.8 \pm 0.3$ hours at $-30^{\circ}$. This can be compared with values reported in the literature (Figure 4.8). The value at $-30^{\circ}$ agrees well with previously reported rotational periods, while the value at $-45^{\circ}$ shows a slightly longer rotation period than fits to Neptune's winds or to the Great Dark Spot. 
Table 4.1 Neptune Observations

\begin{tabular}{|c|c|c|c|c|c|}
\hline$\overline{\text { Date }}$ & Time (UTC) & Object & Filter & Airmass & Seeing \\
\hline \multirow[t]{8}{*}{ Sept. 61996} & $12: 27$ & Neptune & kcont & 2.24 & $0.6^{\prime \prime}$ \\
\hline & $12: 35$ & Neptune & feii & 2.37 & $0.6 "$ \\
\hline & $12: 31$ & Neptune & h210 & 2.31 & $0.6^{\prime \prime}$ \\
\hline & $12: 46$ & FS34 & kcont & 1.8 & \\
\hline & $12: 40$ & FS34 & feii & 1.81 & \\
\hline & $12: 44$ & FS34 & h210 & 1.82 & \\
\hline & $11: 45$ & Neptune & speckle H & 1.80 & \\
\hline & $10: 42$ & SAO 188588 & speckle $H$ & 1.50 & \\
\hline \multirow[t]{7}{*}{ Oct. 101997} & $10: 35$ & Neptune & $\operatorname{ch} 4$ & 2.42 & $1.0 "$ \\
\hline & $10: 39$ & Neptune & h210 & 2.48 & $0.9^{\prime \prime}$ \\
\hline & $10: 43$ & Neptune & feii & 2.63 & $0.8^{\prime \prime}$ \\
\hline & $10: 47$ & Neptune & oii & & $0.9 "$ \\
\hline & $4: 53$ & SJ9105-P530 & $\operatorname{ch} 4$ & 1.5 & \\
\hline & $8: 45$ & Neptune & speckle H & 1.5 & \\
\hline & $8: 32$ & SAO188682 & speckle H & & \\
\hline \multirow[t]{4}{*}{ Oct. 111997} & $9: 15$ & Neptune & $\operatorname{ch} 4$ & 3.7 & $1.8 "$ \\
\hline & $9: 39$ & SJ9101-P525 & $\operatorname{ch} 4$ & 1.0 & \\
\hline & $8: 09$ & Neptune & speckle H & 2.09 & \\
\hline & $8: 09$ & SAO188699 & speckle $H$ & 2.09 & \\
\hline \multirow[t]{4}{*}{ Oct. 121997} & $8: 28$ & Neptune & $\operatorname{ch} 4$ & 2.43 & $0.8^{\prime \prime}$ \\
\hline & $8: 30$ & Neptune & $\operatorname{ch} 4$ & & \\
\hline & $7: 27$ & Neptune & speckle $\mathrm{H}$ & 1.73 & \\
\hline & $6: 15$ & SAO 188682 & speckle $H$ & 1.4 & \\
\hline \multirow[t]{10}{*}{ Oct. 131997} & $6: 12$ & Neptune & $\operatorname{ch} 4$ & 1.40 & $0.6 "$ \\
\hline & $6: 16$ & Neptune & $\mathrm{h} 210$ & 1.42 & $0.45 "$ \\
\hline & $6: 21$ & Neptune & feii & 1.43 & $0.6 "$ \\
\hline & $6: 25$ & Neptune & oii & 1.45 & $0.6^{\prime \prime}$ \\
\hline & $6: 42$ & SJ9182 & $\operatorname{ch} 4$ & 1.15 & \\
\hline & $6: 44$ & SJ9182 & h210 & 1.15 & \\
\hline & $6: 48$ & SJ9182 & feii & & \\
\hline & $6: 52$ & SJ9182 & oii & & \\
\hline & $5: 20$ & Neptune & speckle H & 1.32 & \\
\hline & $5: 39$ & SAO188726 & speckle H & 1.35 & \\
\hline
\end{tabular}




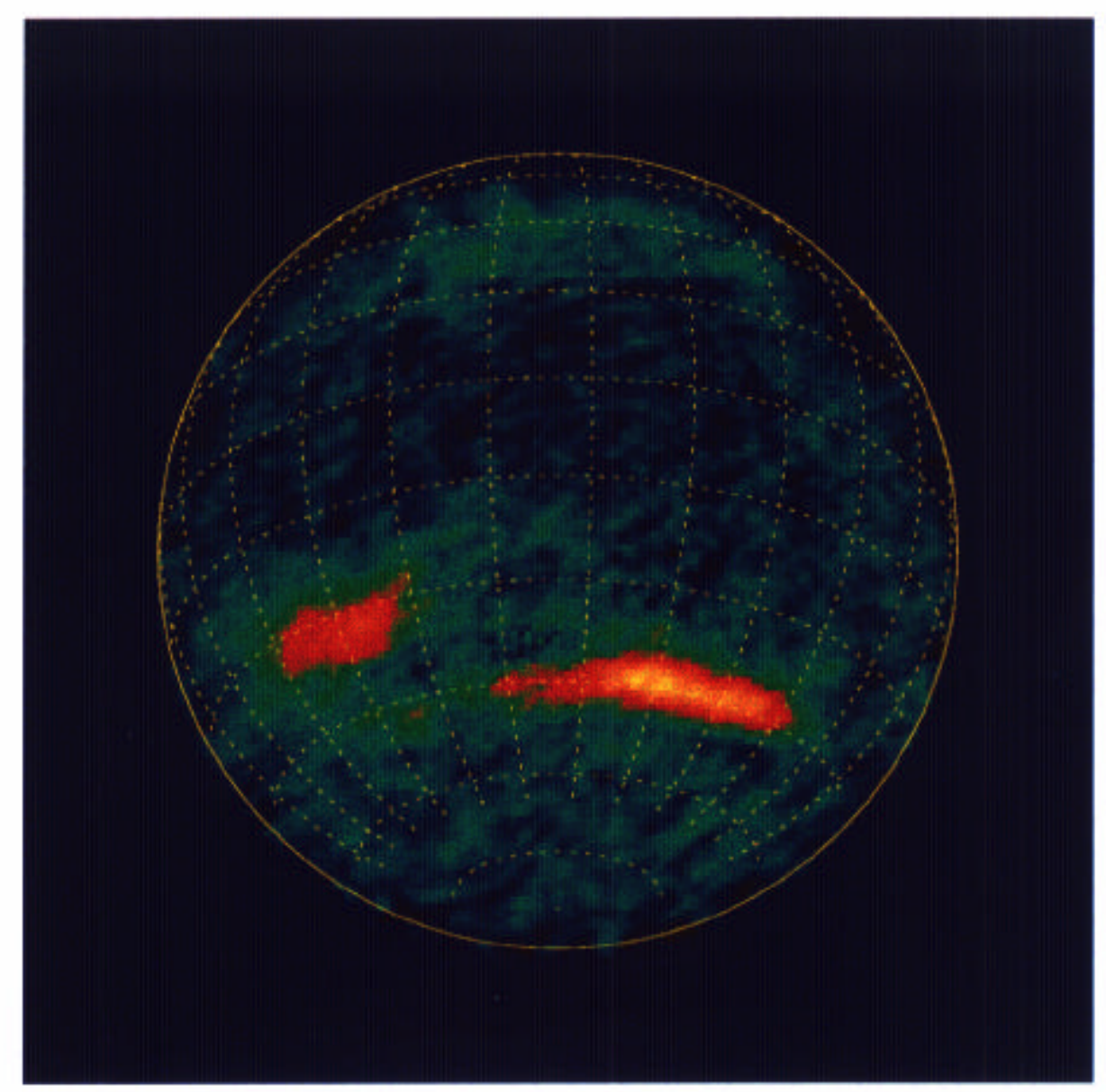

H-band speckle

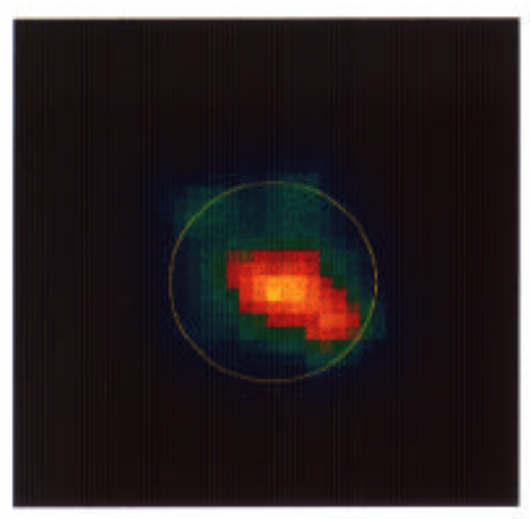

FEII

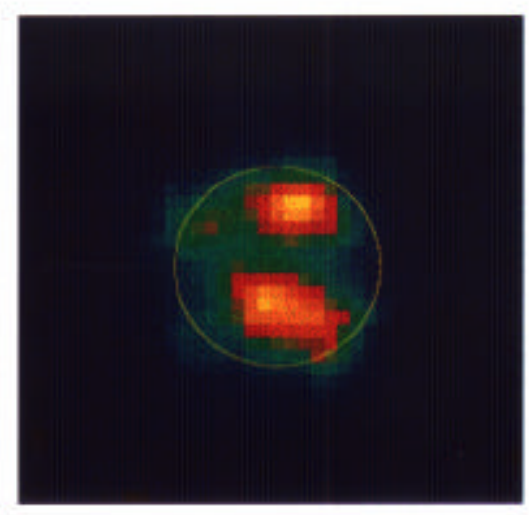

H210

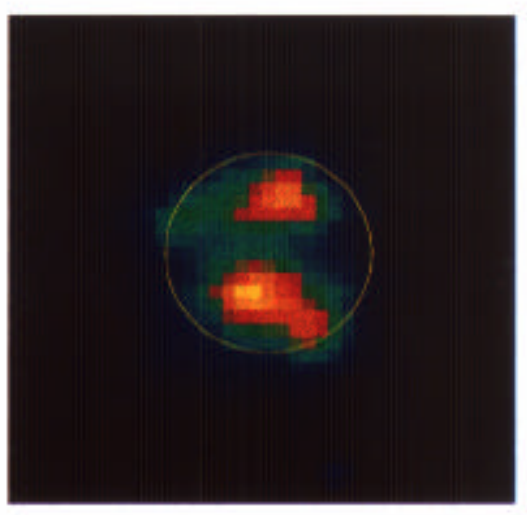

KCONT

Fig. 4.1: Images of Neptune from Sept. 61996 


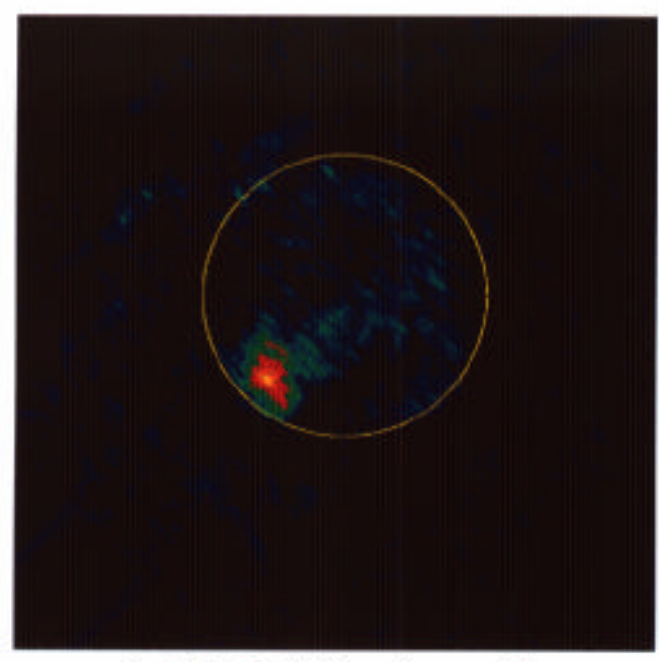

Oct $1097 \mathrm{H}$ band speckle
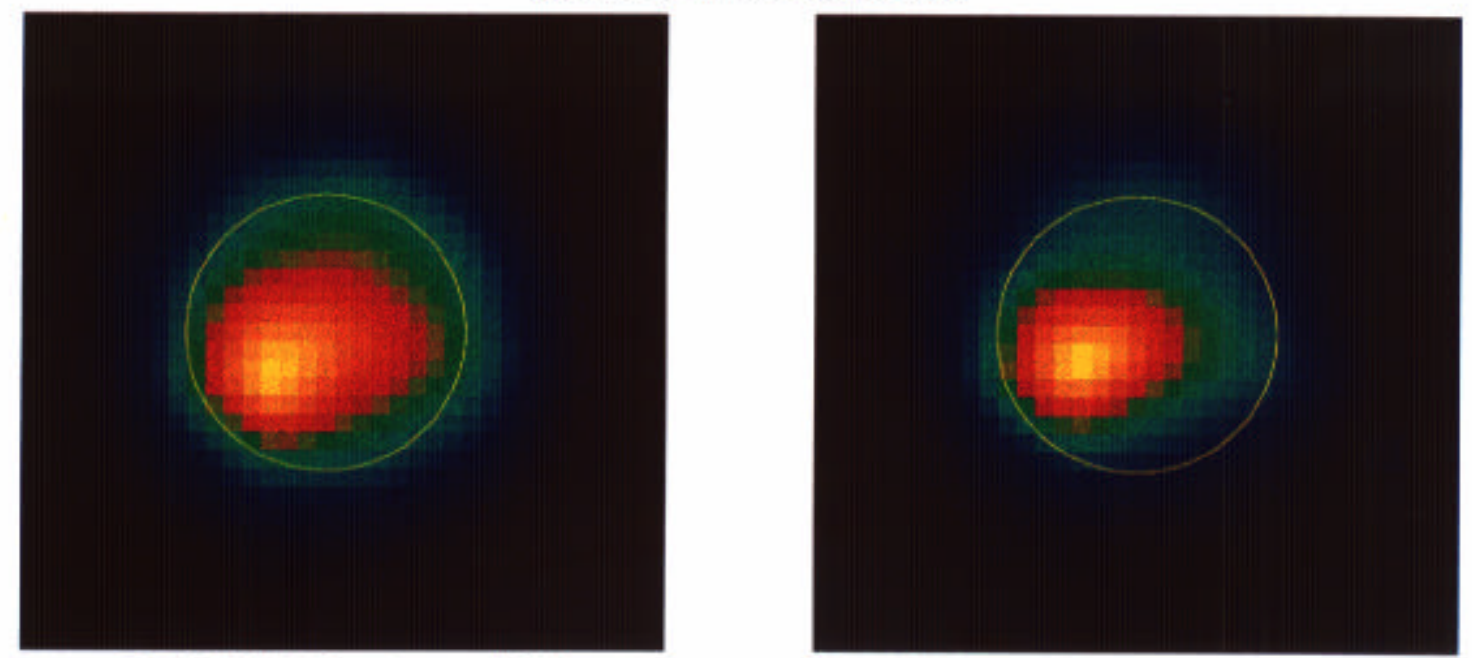

Oct 10971.3 microns (OII)

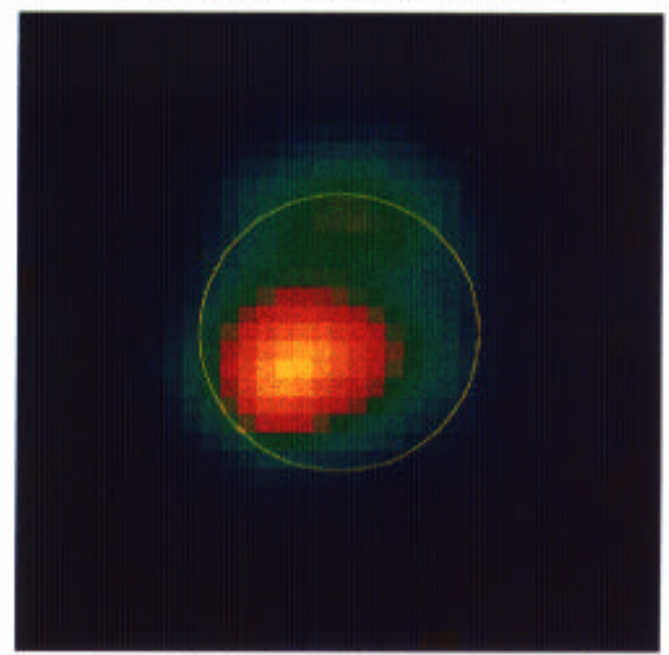

Oct 10972.1 microns (H210)

Oct 10971.6 microns (FEII)

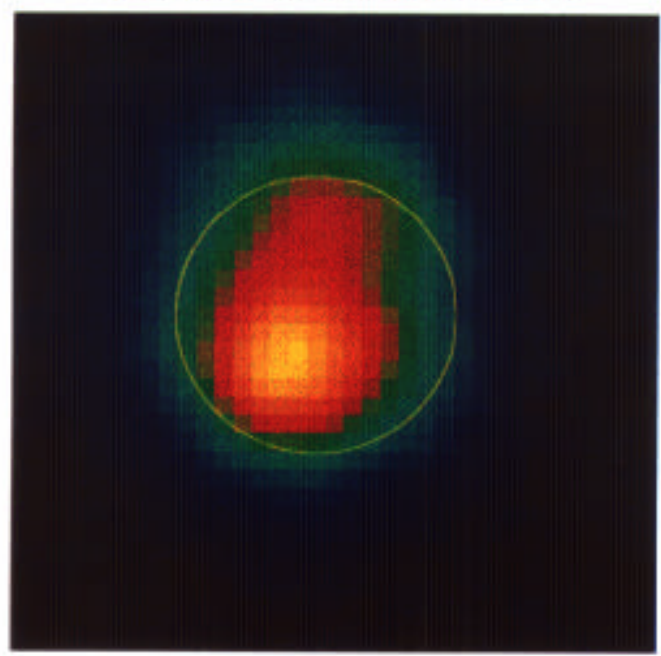

Oct 10972.3 microns $(\mathrm{CH} 4)$

Fig. 4.2: Images of Neptune from Oct. 101997 


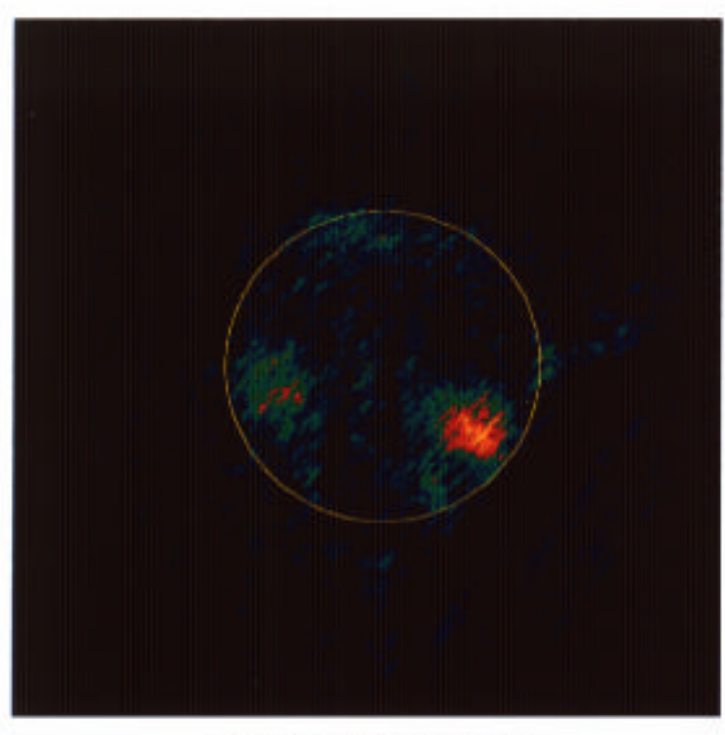

Oct $1197 \mathrm{H}$ band speckle

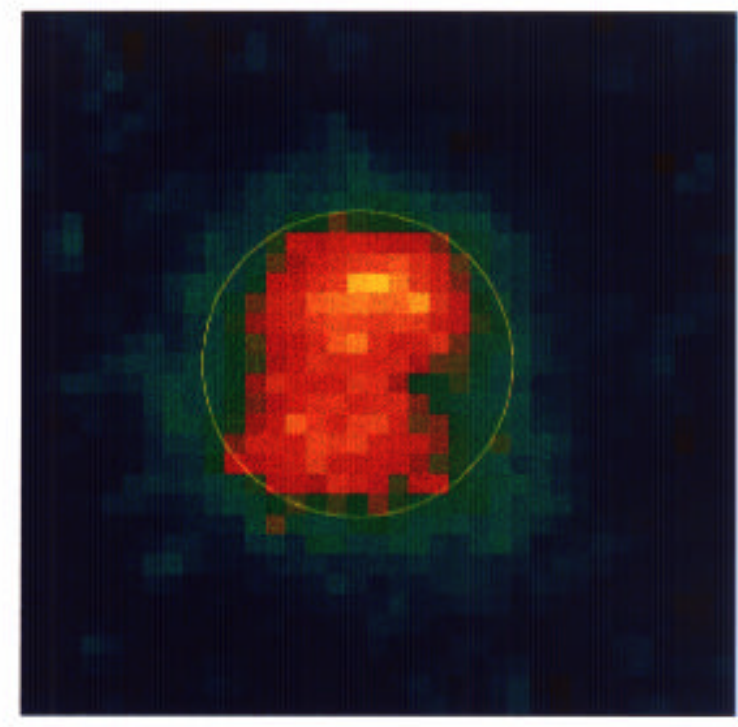

Oct 11972.3 microns (CH4)

Fig. 4.3: Images of Neptune from Oct. 111997 


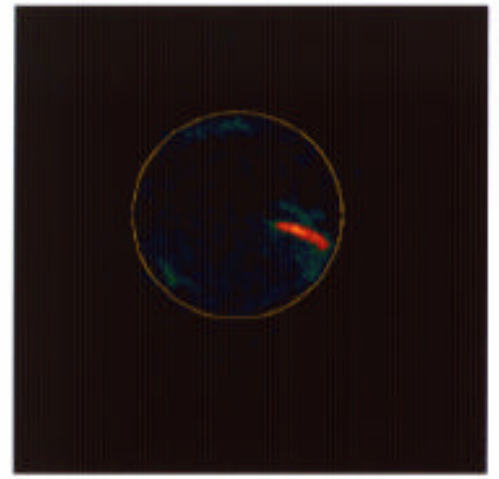

Oct $1297 \mathrm{H}$ band speckle

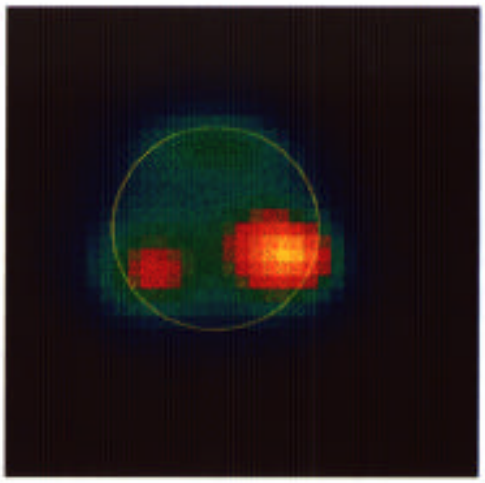

Oct 12971.6 microns (FEII)

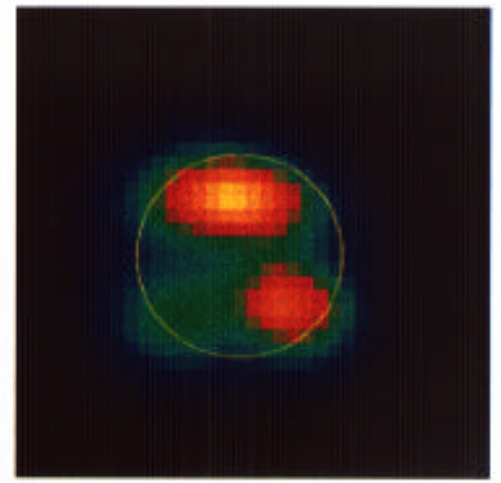

Oct 12972.3 microns (CH4)

Fig. 4.4: Images of Neptune from Oct. 121997 


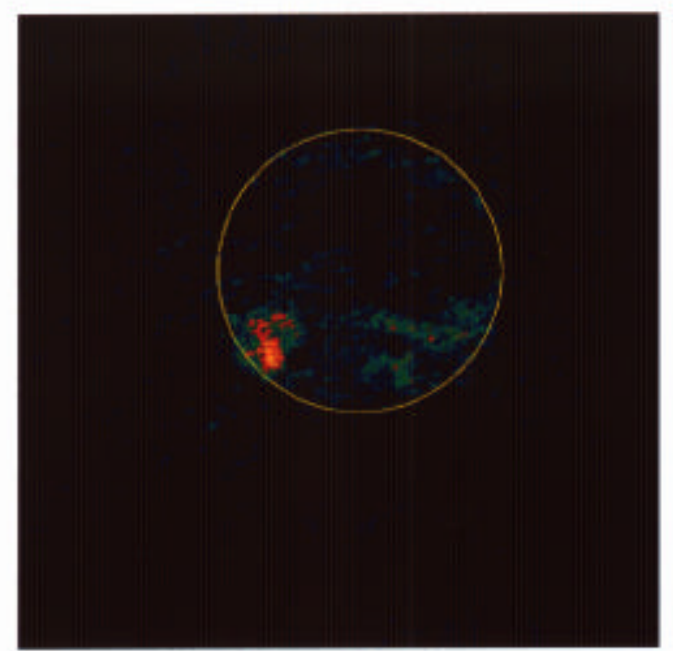

Oct $1397 \mathrm{H}$ band speckle
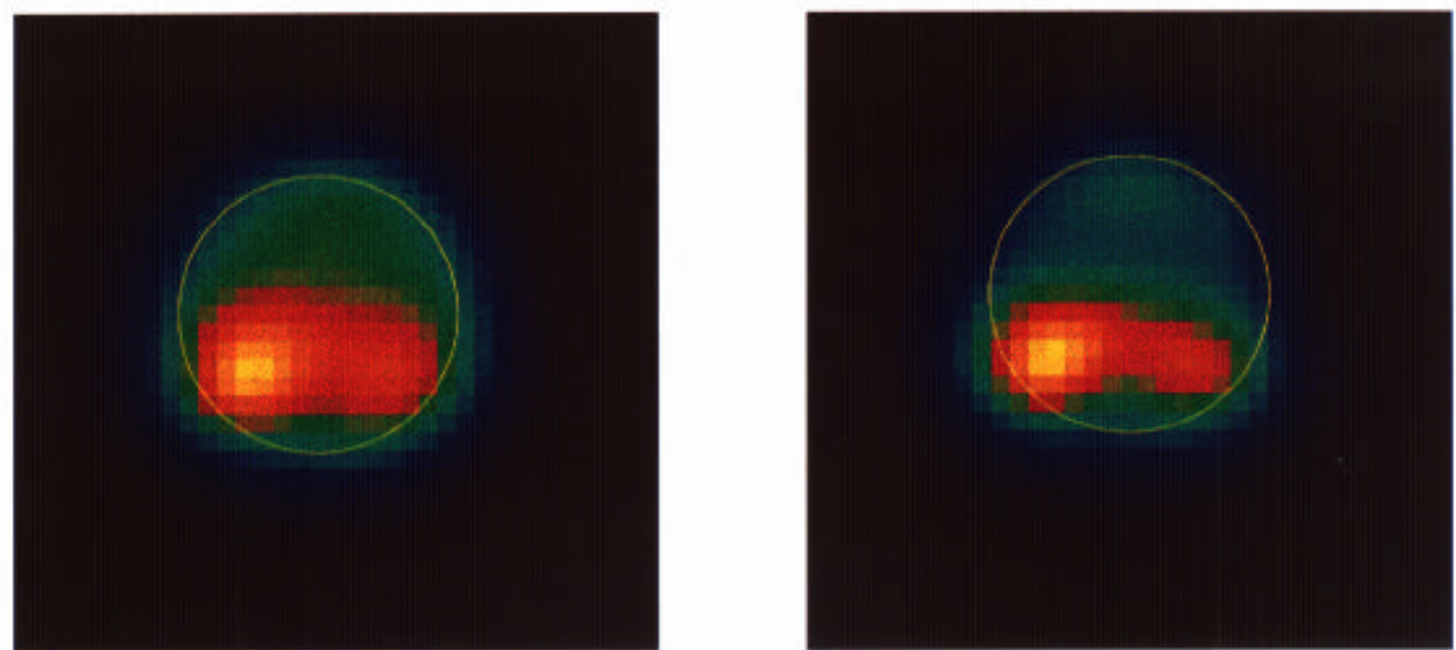

Oct 13971.3 microns (OII)

Oct 13971.6 microns (FEII)

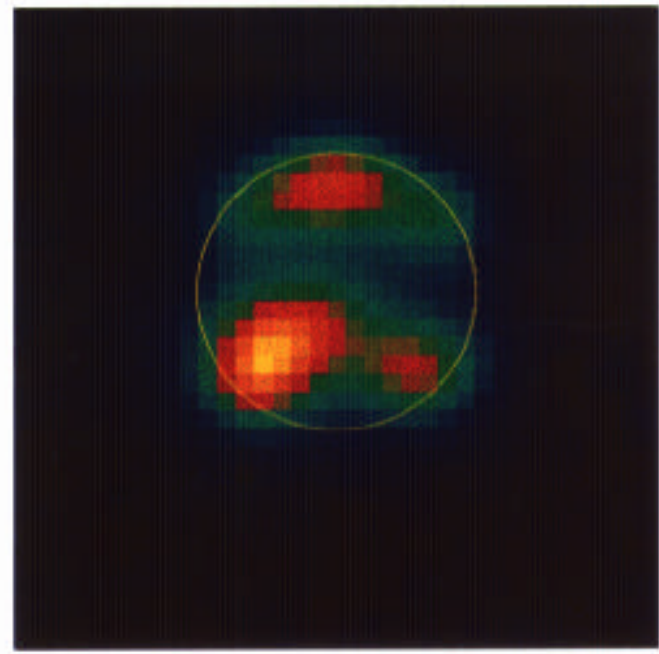

Oct 13972.1 microns (H210)

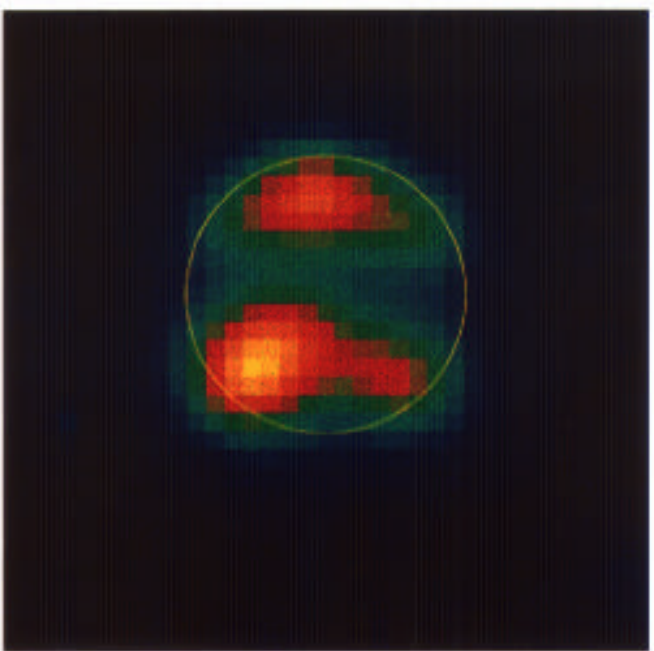

Oct 13972.3 microns $(\mathrm{CH} 4)$

Fig. 4.5: Images of Neptune from Oct. 13 1997. Shown are a speckle Hband image $(4.5 \mathrm{a})$, and four narrownband images $(4.5 \mathrm{~b} 4.5 \mathrm{e})$. These are the images used for the modeling discussed in section 4.3 


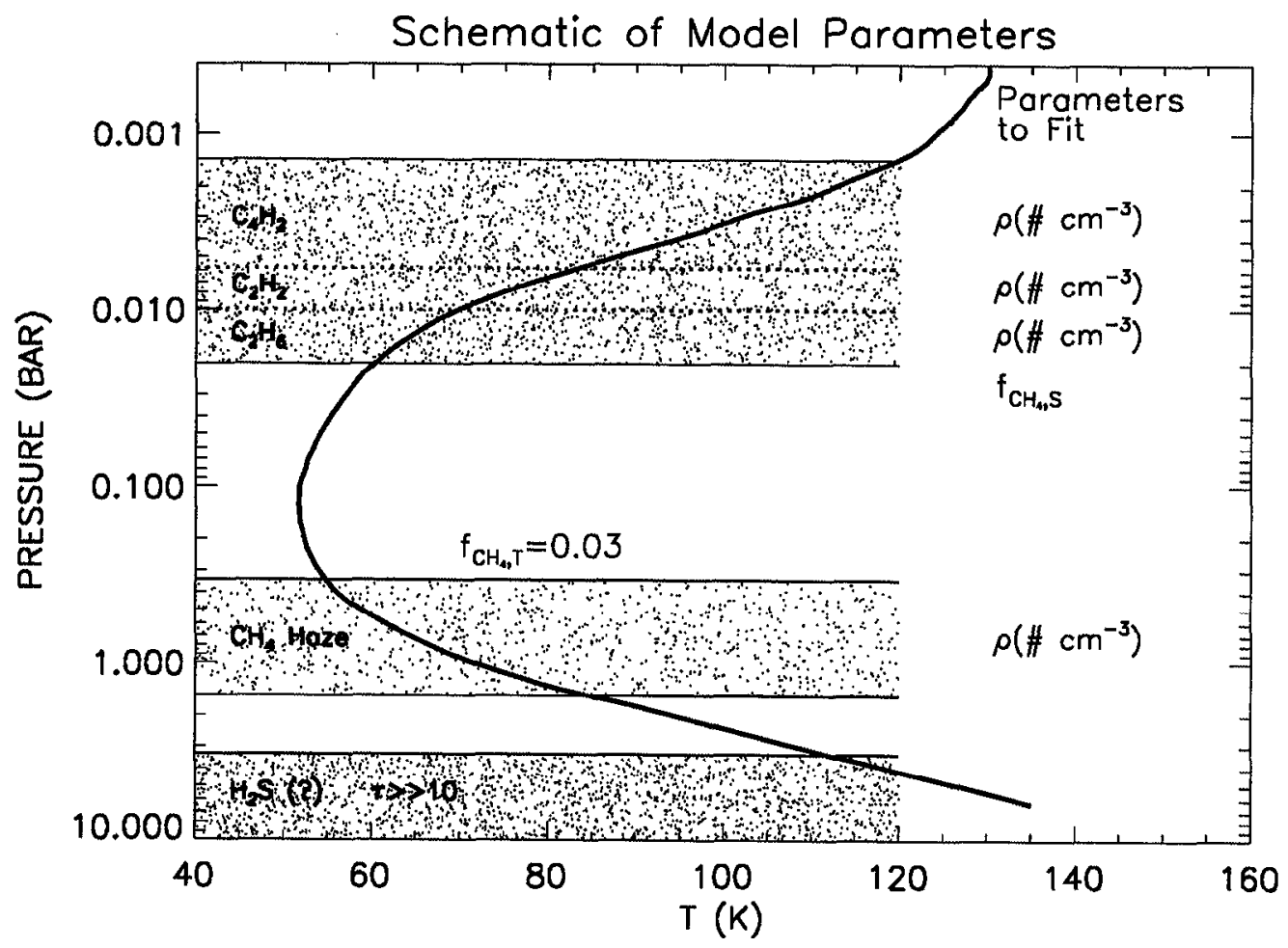

a

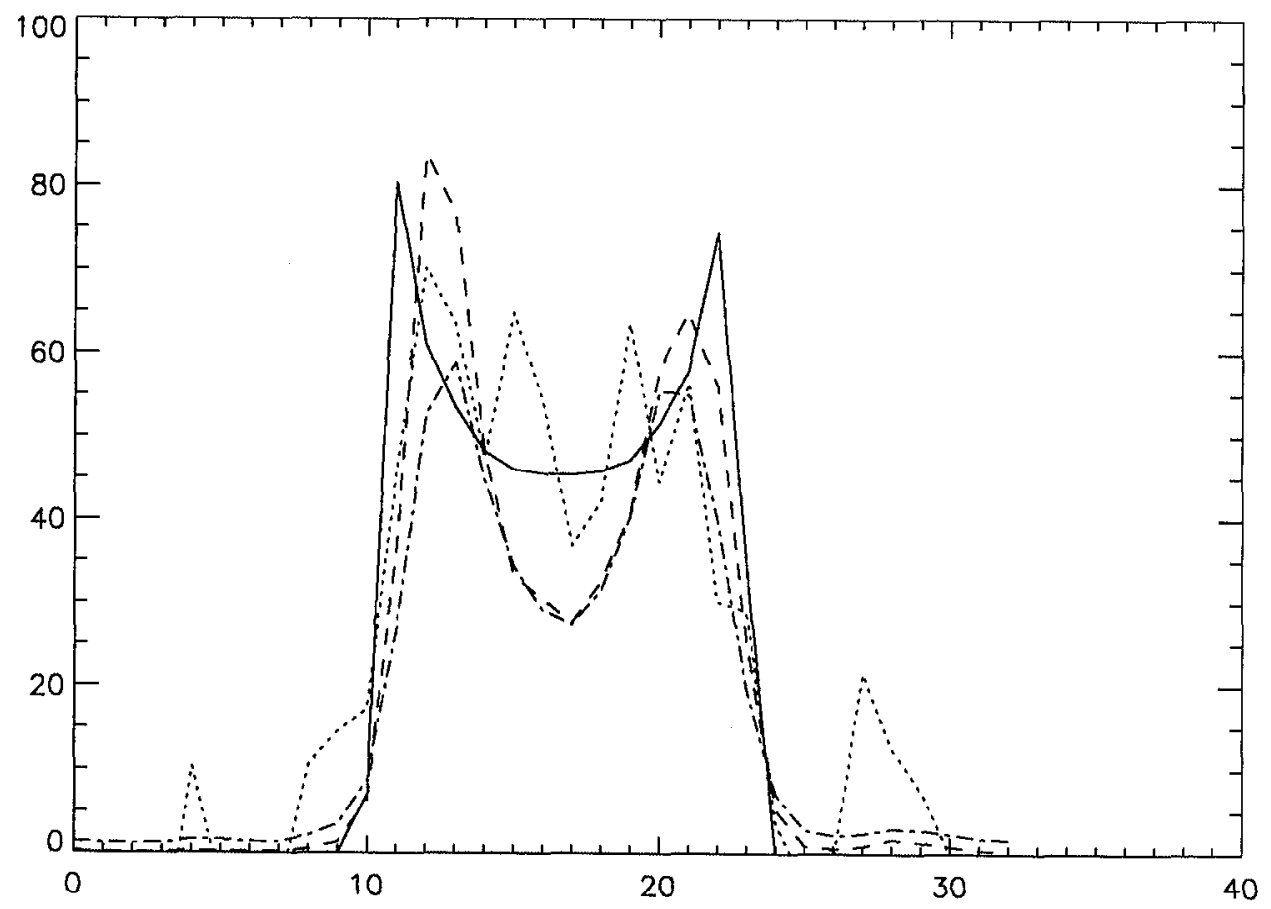

b

Fig. 4.6a: Atmospheric structure. b: scans through center of Neptune 

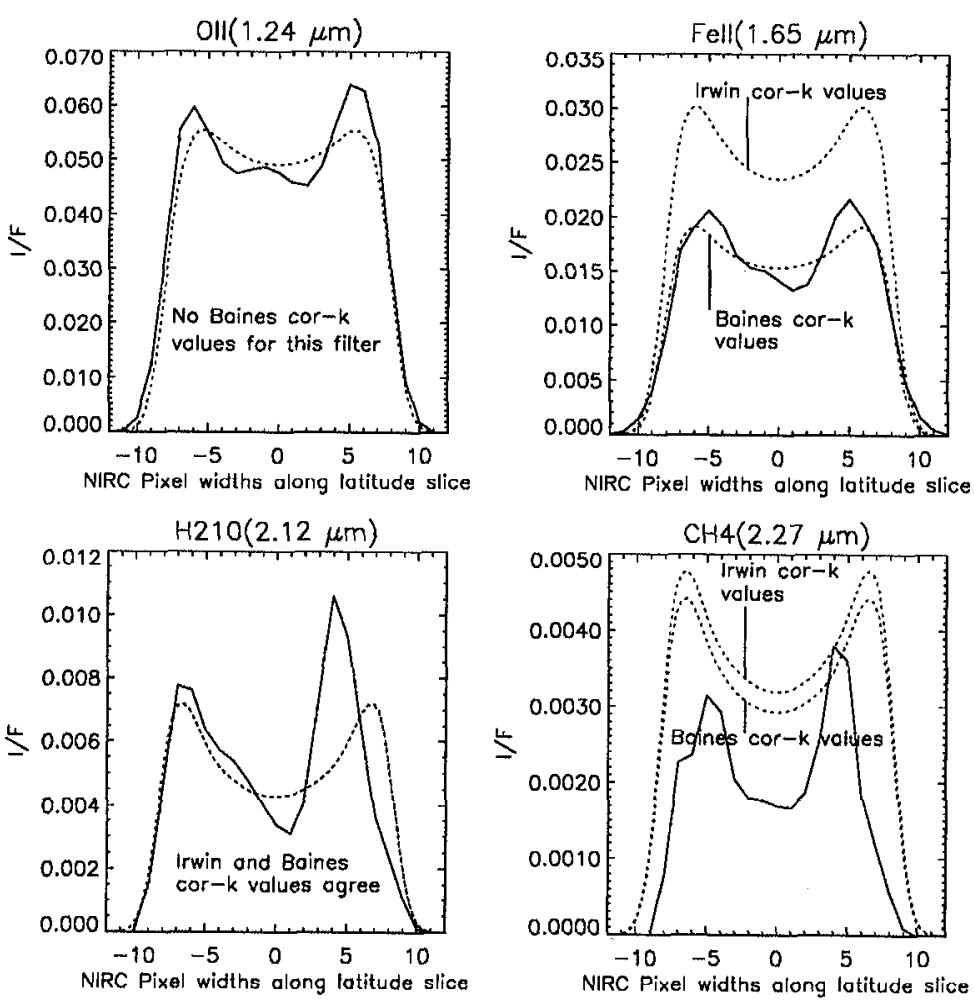

Fig. 4.7: $\mathrm{k}$ distribution coefficient comparison

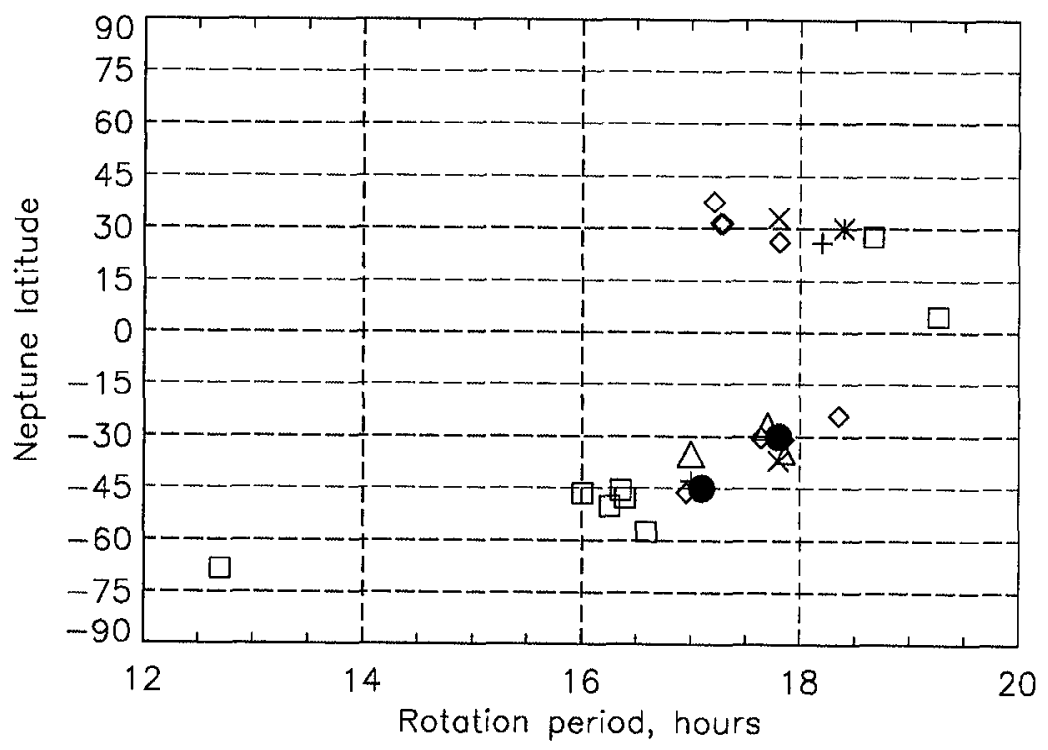

Fig. 4.8: Rotation periods of Neptune at various latitudes as determincd by tracking of cloud features. Dark circles: this work. Plus signs: Roddier et al. 1998. Star: Hammel et al. 1995. Squares: Sromovsky et al. 1995. Diamonds: Hammel and Lockwood 1997. Small trianges: Hammel and Buie 1987. Large triangles: Hammel et al. 1989. X's: Belton and Terrile 1984. 


\section{BIBLIOGRAPHY FOR CHAPTER 4}

Arvesen, J.C., Griffin, R.N. Jr., and Pearson, B.D., Jr. 1969. Determination of extraterrestrial solar spectral irradiance from a research aircraft. Applied Optics 8,22152232 .

Baines, K.H., West, R.A., Giver, L.P., and Moreno, F. 1993. Quasirandom narrowband model fits to nearinfrared lowtemperature laboratory methane spectra and derived exponentialsum absorption coefficients. J. Geophys. Res. 98, 55175529.

Baines, K.H., Hammel, H.B., Rages, K.A., Romani, P.N., and Samuelson, R.E. 1995. Clouds and hazes in the atmosphere of Neptune, in Neptune and Triton, Univ. of Arizona Press, D.P. Cruikshank, ed. p. 489546.

Baines, K.H., and Hammel, H.B., 1994. Clouds, hazes, and stratospheric methane abundance in Neptune. Icarus 109, 2039.

Baines, K.H., and Smith, H.W. 1990. The atmopsheric structure and dynamical properties of Neptune derived from groundbased and CUE spectrophotometry. Icarus 85, 65108 .

Belton, M.J.S., and Terrile, R. 1984. Rotational properites of Uranus and Neptune. in, Uranus and Neptune, J.T. Bergstralh, ed. pp. 327347. NASA CP2330.

Gibbard, S.G., Macintosh, B., Gavel, D., Max. C.E., de Pater, I., Ghez, A.M., Young, E.F., and McKay, C.P. 1999. Titan: highresolution speckle images from the Keck Telescope. Icarus, in press.

Gibbard, S.G., Macintosh, B., Max, C.E., Gavel, D., and de Pater, I. 1998. Highresolution speckle images of Neptune from the Keck Telescope. BAAS 30: 1099. DPS abstract $43 \mathrm{P} 14$.

Hammel, H.B. 1989. Discrete cloud structure on Neptune. Icarus 80, 1422.

Hammel, H.B., Beebe, R.F., de Jong, E.M., Hansen, C.J., Howell, C.D., Ingersoll, A.P., Johnson, T.V., Limaye, S.S., Magalhaes, J.A., Pollack, J.B., Sromovsky, L.A., Suomi, V.E., and Swift, C.E. 1989. Neptune's wind speeds obtained by tracking clouds in Voyager images. Science 245, 13671369.

Hammel, H.B., and Buie, M.W. 1987. An almospheric rotalion period of Neptune deter mined from methaneband imaging. Icarus $72,6268$.

Hammel, H.B., and Lockwood, G.W. 1997. Atmospheric structure of Neptune in 1994, 1995, and 1996: HST imaging at multiple wavelengths. Icarus, 129, 466481. 11

Hammel, H.B., Lockwood, G.W., Mills, J.R., and Barnet, C.D. 1995. Hubble Space Telescope imaging of Neptume's cloud structure in 1994. Science 268, 17402.

Hammel, H.B., Lawson, S.L., Harrington, J., Lockwood, G.W., Thompson, D.T., and Swift, C. 1992. An atmospheric outburst on Neptune from 1986 through 
1989. Icarus $99,363367$.

Ingersoll, A.P., Barnet, C.D., Beebe, R.F., Flasar, F.M., Hinson, D.P., Limay e, S.S., Sromosky, L.A., and Suomi, V.Y., 1995. Dynamic metereology of Neptune, in Neptune and Triton, Univ. of Arizona Press, D.P. Cruikshank, ed., p. 613682.

Irwin, P.G J., Calcutt, S.B., Taylor, F.W., and Weir, A.L. 1996. Calculated k distribution coefficients for hydrogen and selfbroadened methane in the range 20009500 $\mathrm{cm}^{-1}$ from exponential sum fitting to band-modeled spectra. J. Geophys. Res. 101, 26137-26154.

Lockwood, G.W., Thompson, D.T., Hammel, G.B., Birch, P., and Candy, M. 1991. Neptune's cloud structure in 1989: photometric variations and correlation with groundbased images. Icarus 90, 299-307.

Lohmann, A., G. Weigelt, and B. Wirnitzer. 1983. Applied Optics 22, 4028-4037.

Matthews, K., and B.T. Soifer. 1994. Infrared Astronomy with Arrays: the Next Generation I. McLean, ed. (Dordrecht: Kluwer Academic Publishers), p. 239.

Pearl, J.C., and B.J. Conrath. 1991. The albedo, effective temperature, and energy balance of Neptune, as determined from Voyager data. Geophys. Res. Lett. 96, 18921-18930.

Roddier, F., Roddier, C., Graves, J.E., Northcott, M.J., and Owen, T. 1998. NOTE: Neptune's cloud structure and activity: groundbased monitoring with adaptive optics. Icarus 136, 168172.

Roddier, F., C. Roddier, A. Brahic, C. Dumas, J.E. Graves, M.J. Northcott, and T. Owen. 1997. First groundbased adaptive optics observations of Neptune and Proteus. Planetary and Space Science 45, 10311036. 12

Romani, P.N., and Atreya, S.K. 1989. Stratospheric aerosols from $\mathrm{CH}_{4}$ photochemistry on Neptune. Geophys. Res. Lett. 16, 941944.

Romani, P.N., Bishop, J., Bezard, B., and Atreya, S. 1993. Methane photochemistry on Neptune: methane and acetylene mixing ratios and haze production. Icarus $106,442463$.

Smith, B.A., and 64 others. 1989. Voyager 2 at Neptune: imaging science results. Science 246, 14221449.

Sromovsky, L.A., Limaye, S.S., and Fry, P.M. 1995. Clouds and circulation on Neptune: implications of 1991 HST observations. Icarus 118, 2538.

Sromovsky, L.A., Limaye, S.S., and Fry, P.M. 1993. Dynamics of Neptune's major cloud features. Icarus 105, 110141. 


\section{VOLCANOES ON IO}

Here we present preliminary results of near-infrared observations of the Galilean moon Io in 1998 with speckle interferometry. These observations have a resolution of $60 \mathrm{~km} / \mathrm{pixel}$, greater than any instrument except Galileo's SSI. Observations from July 12 1998, July 28 1998, and August 41998 show 18 distinct hotspots (see Figure 5.1). 17 are present in the July 12 image, with a bright transient visible only on July 28 - August 4. On July 12 Loki was fading from an outburst event, and the position of Loki appears to shift between July 12 and August 4.

In the best data set (Jul 12 1998) 17 distinct hotspots were detected, the largest number detected by any group in a single Io image. Nearly all either align with known Io features or repeat in the July 28 or August 4th data. Sources span a factor of ten in intensity.

Source positions have been registered on Io's disk, including corrections for distortions in the camera optics and uncertainties in the camera rotation to an absolute accuracy of ten miliarcseconds (see Table 5.2). Images were registered with Io's disk on the basis of the Galileo SSI position of Janus (given in McEwen et al 1998) Images are rotated so that Io's north pole is up, and corrected for slight NIRC field distortions The single largest error term is in fact the uncertainty in Galileo visible-light measurements used for reference.

Loki, the brightest source on this hemisphere, was observed fading from a major transient. There is some evidence that it's position shifted as it faded, indicating that the transient event occured in a different part of Loki's horseshoe-shaped caldera than the main event (Figure 5.2). Loki may in fact be a cluster of seperate hotspots associated within one volcanic shield, similar to Hawail's Mauna Loa volcano.

Temperature measurements based on multiple wavelengths are consistent with hightemperature silicate vulcanism. We successfully imaged Io at mid-IR ( 3 micron) wavelengths using Keck's chopping secondary, the first Keck speckle observations to use this mode.

Another source worth noting is source 6. Its latitude is consistent with Pele, a major volcanic region. However, Pele was located just behind Io's limb during these observations and it should (barely) have not been visible. We may be seeing the IR glow from Pele reflected of its volcanic plume - if confirmed this will constrain the composition of the plume.

The sources - excluding Loki - are relatively uniformly distributed on Io's surface (at weak statistical significance.) This is somewhat different than the distribution seen by Galileo, but Galileo's SSI is biased towards the hottest events. Uniform distribution of sources would favour a nearly solid Io as opposed to a partially molten asthenosphere. 
Table 5.1: Io observations.

\begin{tabular}{|c|c|c|}
\hline $\begin{array}{c}\text { Date \& } \\
\text { Time }\end{array}$ & $\begin{array}{c}\text { Wavelength } \\
(\mu \mathrm{m})\end{array}$ & Number of Frames \\
\hline 12 July 1998 & $\mathrm{K}(2.2)$ & $8 \times 100$ \\
\cline { 2 - 3 } 12:10-12:55 UT & $\mathrm{H}(1.7)$ & $4 \times 100$ \\
\hline 28 July 1998 & $\mathrm{K}(2.2)$ & $4 \times 100$ \\
\cline { 2 - 3 } 10:25-11:31 UT & $\mathrm{nbL}(3.0)$ & $6 \times 40$ \\
\hline 4 Aug. 1998 & $\mathrm{~K}(2.2)$ & $6 \times 100$ \\
\cline { 2 - 3 } 12:15-13:17 & $\mathrm{nbL}(3.0)$ & $6 \times 40$ \\
\hline
\end{tabular}

Table 5.2: Uncertainty in the placement of hotspots in our Io observations. For Loki, These translate into a $\left(0.82^{\circ}, 0.94^{\circ}\right)$ uncertainty in latitude and longitude.

\begin{tabular}{|c|c|c|}
\hline Error Source & $\begin{array}{c}\text { X error } \\
\text { (pix) }\end{array}$ & $\begin{array}{c}\text { Y error } \\
\text { (pix) }\end{array}$ \\
\hline Intrinsic centroiding error & 0.20 & 0.20 \\
\hline Error in Janus centroid & 0.20 & 0.20 \\
\hline $0.2^{0}$ uncertainty in camera orientation & 0.02 & 0.13 \\
\hline $0.5 \%$ uncertainty in plate scale & 0.18 & 0.03 \\
\hline Io deviation from sphericity & 0.18 & 0.03 \\
\hline Uncertainty in camera distortion & 0.1 & 0.1 \\
\hline Uncertainty in SSI Janus position & 0.25 & 0.25 \\
\hline Total & 0.47 & 0.42 \\
\hline
\end{tabular}


Table 5.3: Source fluxes, in gigawatts/ $\mu \mathrm{m} / \mathrm{steradian}$

\begin{tabular}{|c|c|c|c|}
\hline Source & 12 July Flux & 28 July Flux & 4 Aug. Flux \\
\hline 0 (Janus) & 1.07 & 0.9 & 0.71 \\
\hline 1 (Kan.) & 0.53 & 0.5 & 0.46 \\
\hline 2 (Loki) & 8.18 & 5.0 & 4.79 \\
\hline 3 & 0.49 & 0.5 & 0.25 \\
\hline 4 (Fjorgyn) & 0.20 & & 0.21 \\
\hline 5 (Acala) & 0.63 & & 0.55 \\
\hline 6 (Pele?) & 0.36 & & 0.13 \\
\hline 8 & 0.13 & & \\
\hline 10 (Surt) & 0.15 & & \\
\hline 11 & 0.20 & & 0.29 \\
\hline 12 (Fuchi) & 0.23 & & 0.15 \\
\hline 13 (Ra) & 0.14 & & 0.12 \\
\hline 14 & 0.15 & & 0.13 \\
\hline 15 & 0.18 & & 1.48 \\
\hline 16 (Aten) & 0.25 & & \\
\hline 17 & 0.23 & & 3.93 \\
\hline 18 & & & \\
\hline
\end{tabular}




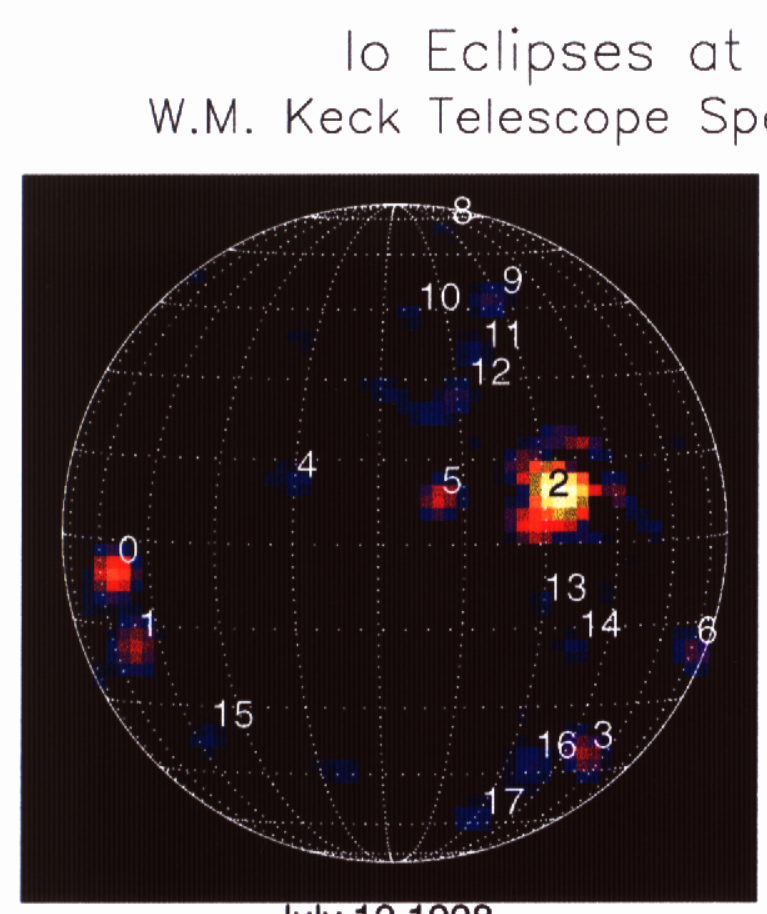

July 121998

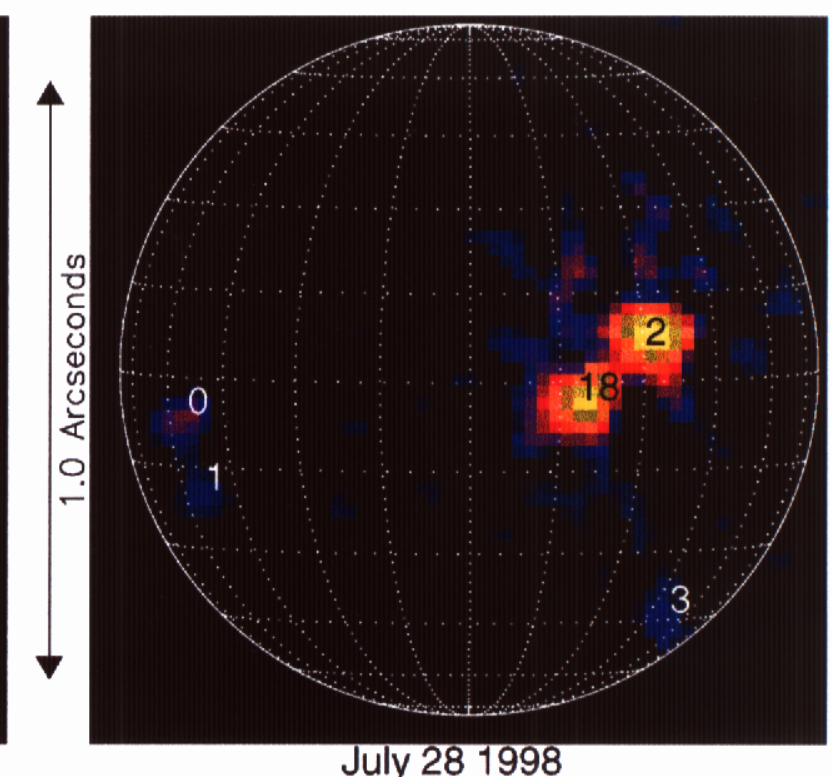

July 281998

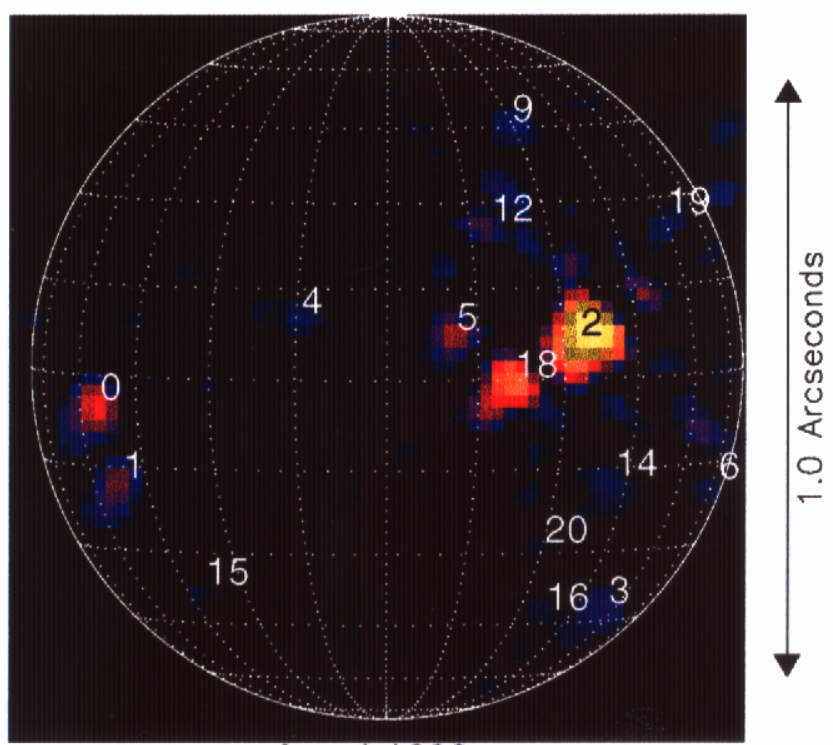

All images displayed with logrithmic intensity scale.

B. Macintosh, C. Max, D. Gavel, S. Gibbard, LLNL I. de Pater, ÚC Berkeley, A. Ghez, UCLA

Aug 41998

Figure 5.1 Io volcanic hotspots identified in Keck speckle data. 


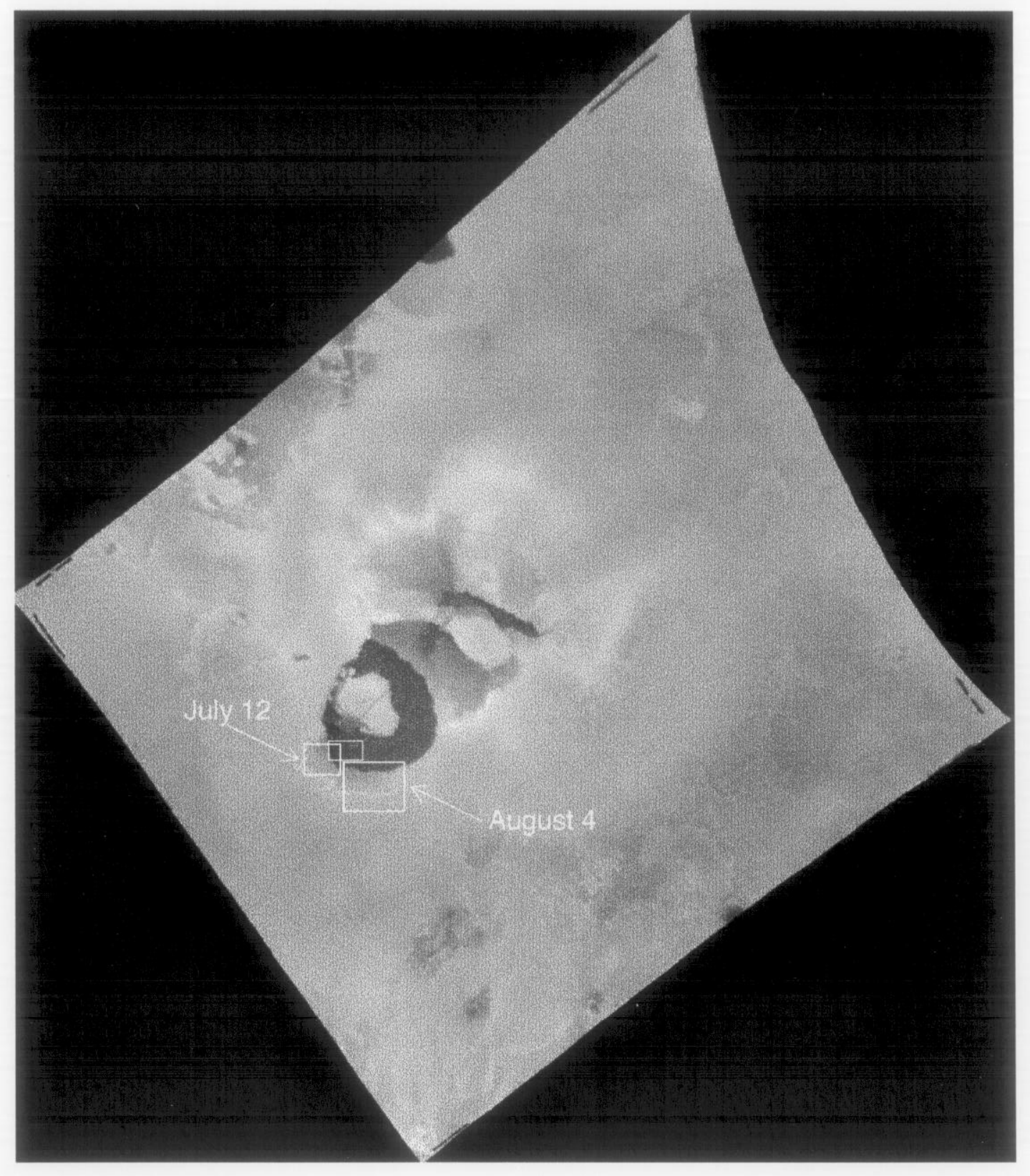

Figure 5.2 The Loki hot spot apparently shifted during the July 12 to August 4, 1998 time period. Error boxes for our determination of hot spot centers are overlayed on a Voyager image of the region. The unlabled box is a Galileo Solid-State Images (SSI) determination, from McEwan, et. al., 1998. 
B. Macintosh, D. Gavel, S. Gibbard, C.E. Max, I. de Pater, A. Ghez, M. Eckart, 1998. Infrared Speckle Imaging of Io's Volcanoes with $60 \mathrm{~km} /$ pixel Resolution, American Geophysical Union Fall Meeting, San Francisco, CA.

McEwen,AlfredS., Keszthelyi,Laszlo, Geissler,Paul, Simonelli,DamonP., Carr,MichaelH., Johnson, TorrenceV., Klaasen,KennethP., Breneman,H.Herbert, Jones,ToddJ., Kaufman,JamesM., Magee,KariP., Senske,DavidA., Belton,MichaelJ.S., Schubert,Gerald, 1998. Active Volcanism on Io as Seen by Galileo SSI Icarus, Volume 135, 181-219. 


\section{HIGH RESOLUTION SOLAR SYSTEM ASTRONOMY: THE FUTURE:}

Speckle interferometry has allowed us to collect new scientific data on the solar system objects Titan, Neptune, and Io. However, a fundamental physical requirement, the need to gather enough light during each atmospheric turnover time to detect the object, limits the application of speckle interfcrometry to only the very brightest of astronomy targets, and (in the struggle to gather enough light) requires observing over very broad wavelength bands.

Adaptive optics, with laser beacons as artificial guide stars, will overcome these limitations. Long exposure observations of dim objects in narrow wavelength bands will simply: be a matter of pointing the laser at the target, turning on the adaptive optics wavefront control loops, and opening the shutter. Adaptive optics at the Keck 10 meter telescope, a project in which the Lawrence Livermore National Laboratory has been heavily involved, has seen its "first light" in February 1999. The laser beacon is due to follow later this year. See Gavel, 1994 for a predictive performance analysis of the Keck adaptive optic system.

With adaptive optics enabling long exposures and narrow wavelength band imaging at high resolution, a number of interesting planetary science questions can be addressed. It will be possible to probe Titan's atmosphere more directly, for example by imaging both inside and outside the Methane absorption bands. With the grism spectrometer in the second generation Keck infrared camera, NIRC-II, it will be possible to do spectral mapping of interesting portions of Titan's surface, for example the bright highland areas or the dark "lake" regions. Spectral profiles can provide direct clues to the chemical composition, distinguishing between rock, ice, or hydrocarbons for example. This will help answer some very fundamental questions directly. Neptune's cloud layers can be mapped at both high spatial and vertical resolution giving a better understanding of the physical processes in the troposphere and stratosphere. Exciting new possibilities open up with dimer solar system objects: for example studying the structure of Uranus' rings or studying the composition and shape of asteroids and their possible companion moonlets.

Certainly also, extra-solar system and extra-galactic astronomy will be profoundly affected by adaptive optics. Since discussion of these topics is beyond the scope of this report, we direct the interested reader to the excellent science justification write up in the plans for the Keck adaptive optics system (Nelson, 1994). 


\section{BIBIJOGRAPHY FOR CHAPTER 6}

Gavel, D.T., J.R. Morris, R. Vernon, 1994, Systematic Analysis of Laser Guide Star Adaptive Optics Systems for Large Telescopes, JOSA-A 11, 2, pp 914-924.

Nelson, J., et. al., 1994, Adaptive Optics for Keck Observatory, Keck Adaptive Optics Note \#208, Chapter 2. 